\title{
The Scope of Ru(II)-Catalyzed Synthesis of Pyridines from Alkynes and Nitriles
}

\author{
Jesús A. Varela, Luis Castedo and Carlos Saá* \\ Departamento de Química Orgánica y Unidad Asociada al CSIC, Facultad de Química, Universidad de \\ Santiago de Compostela, 15782 Santiago de Compostela, Spain
}

\section{Supporting Information}

\section{Contents}

I. General

II. Preparation of starting materials and catalysts

III. General procedures for the Ru-catalyzed $[2+2+2]$ cycloadditions

IV. References 


\section{General}

Dry DMF (Fluka) was used as received. All other solvents and reagents were used as received, unless otherwise noted. All reactions were carried out under an atmosphere of argon in flame-dried glassware with magnetic stirring, unless otherwise indicated.

\section{Preparation of starting materials and catalysts}

2,2-Diprop-2-ynyl malonic acid dimethyl ester (2a) was prepared following published procedures. ${ }^{1}$ Catalysts $\left[\mathrm{CpRu}\left(\mathrm{CH}_{3} \mathrm{CN}\right)_{3}\right] \mathrm{PF}_{6}$ and $\left[\mathrm{Cp} * \mathrm{Ru}\left(\mathrm{CH}_{3} \mathrm{CN}\right)_{3}\right] \mathrm{PF}_{6}$ were also prepared following published procedures., ${ }^{2,3}$

\section{General procedures for the Ru-catalyzed $[2+2+2]$ cycloadditions:}

3-Cyanomethyl-5,7-dihydro-[2]pyridine-6,6-dicarboxylic acid dimethyl ester (4r). In a $25 \mathrm{~mL}$ round-bottomed flask was prepared, under argon, a solution of $\left[\mathrm{Cp} * \mathrm{Ru}\left(\mathrm{CH}_{3} \mathrm{CN}\right)_{3}\right] \mathrm{PF}_{6}(25 \mathrm{mg}, 10 \%)$ and $\mathrm{Et}_{4} \mathrm{NCl}(8 \mathrm{mg}, 10 \%)$ in $\mathrm{DMF}$ (3 mL). After stirring for 10 minutes at room temperature the two partners were successively added, the nitrile $3 \mathbf{r}$ (66 mg, $1 \mathrm{mmol}, 2$ eq.) and then, after 10 minutes, the diyne $2 \mathbf{a}$ (104 $\mathrm{mg}, 0.5 \mathrm{mmol}, 1$ eq.). Stirring was continued for another $10 \mathrm{~min}$ and the reaction was quenched with saturated aqueous solution of $\mathrm{NH}_{4} \mathrm{Cl}(10 \mathrm{~mL})$ and extracted with ethyl ether $(3 \mathrm{x}$ $10 \mathrm{~mL}$ ). The organic layers were collected, dried over anhydrous $\mathrm{Na}_{2} \mathrm{SO}_{4}$ and evaporated under vacuum. Column chromatography of the residue on silica gel using ethyl acetate/hexane (1:1) as eluents afforded pyridine $\mathbf{4 r}$ in $84 \%$ yield. Similar results were obtained when using lower or higher concentration of starting materials.

Pyridine-2,3,6-tricarboxylic acid trimethyl ester (13ax). In a $25 \mathrm{~mL}$ round-bottomed flask was prepared, under argon, a solution of $\left[\mathrm{Cp}^{*} \mathrm{Ru}\left(\mathrm{CH}_{3} \mathrm{CN}\right)_{3}\right] \mathrm{PF}_{6}(25 \mathrm{mg}, 5 \%)$ and $\mathrm{Et}_{4} \mathrm{NCl}(8 \mathrm{mg}, 5 \%)$ in DMF ( $3 \mathrm{~mL}$ ). After stirring for 10 minutes at room temperature the two partners were successively added, the nitrile $3 \mathbf{x}$ ( $85 \mathrm{mg}, 0.08 \mathrm{~mL}, 1 \mathrm{mmol}, 1$ eq.) and then, after 10 minutes, the alkyne $11 \mathbf{a}$ ( 84 $\mathrm{mg}, 0.09 \mathrm{~mL}, 1 \mathrm{mmol}, 1$ eq.). Stirring was continued for $12 \mathrm{~h}$ and the reaction was quenched with saturated aqueous solution of $\mathrm{NH}_{4} \mathrm{Cl}(10 \mathrm{~mL})$ and extracted with ethyl ether $(3 \mathrm{x} 10 \mathrm{~mL})$. The organic layers were collected, dried over anhydrous $\mathrm{Na}_{2} \mathrm{SO}_{4}$ and evaporated under vacuum. Column chromatography of the residue on silica gel using ethyl acetate/hexane (4:6) as eluents afforded pyridine 13ax in $82 \%$ yield.

3,6-Bistrifluoromethylpyridine-2-carboxylic acid methyl ester (13bx): In a $25 \mathrm{~mL}$ round-bottomed flask was prepared, under argon, a solution of $\left[\mathrm{Cp}^{*} \mathrm{Ru}\left(\mathrm{CH}_{3} \mathrm{CN}\right)_{3}\right] \mathrm{PF}_{6}(25 \mathrm{mg}, 5 \%)$ and $\mathrm{Et}_{4} \mathrm{NCl}(8$ $\mathrm{mg}, 5 \%)$ in DMF (3 mL). After stirring for 10 minutes at room temperature the two partners were successively added, the nitrile $3 \mathbf{x}(85 \mathrm{mg}, 0.08 \mathrm{~mL}, 1 \mathrm{mmol}, 1$ eq. $)$ and then, after 10 minutes, excess alkyne 11b was bubbled into the solution. Stirring was continued for $12 \mathrm{~h}$ and the reaction was quenched with saturated aqueous solution of $\mathrm{NH}_{4} \mathrm{Cl}(10 \mathrm{~mL})$ and extracted with ethyl ether $(3 \mathrm{x}$ $10 \mathrm{~mL}$ ). The organic layers were collected, dried over anhydrous $\mathrm{Na}_{2} \mathrm{SO}_{4}$ and evaporated under vacuum to afforded $\mathbf{1 3 b x}$ in $20 \%$ yield.

\section{3-Ethylsulfanyl-5,7-dihydro-[2]pyridine-6,6-dicarboxylic acid dimethyl ester (4e)}

${ }^{1} \mathrm{H}-\mathrm{NMR}\left(250 \mathrm{MHz}, \mathrm{CDCl}_{3}\right) \delta(\mathrm{ppm}): 8.23(\mathrm{~s}, 1 \mathrm{H}), 7.01(\mathrm{~s}, 1 \mathrm{H}), 3.72(\mathrm{~s}, 6 \mathrm{H}), 3.52(\mathrm{~s}, 2 \mathrm{H}), 3.49(\mathrm{~s}$, $2 \mathrm{H}), 3.10$ (c, J=7.3 Hz, 2H), $1.32(\mathrm{t}, \mathrm{J}=7.3 \mathrm{~Hz}, 3 \mathrm{H}) .{ }^{13} \mathrm{C}-\mathrm{NMR}$, DEPT $\left(63 \mathrm{MHz}, \mathrm{CDCl}_{3}\right) \delta(\mathrm{ppm})$ : $171.3(2 \times \mathrm{C}), 157.4(\mathrm{C}), 149.9(\mathrm{C}), 144.8(\mathrm{CH}), 132.1(\mathrm{C}), 117.6(\mathrm{CH}), 60.1(\mathrm{C}), 53.1\left(2 \mathrm{x} \mathrm{CH}_{3}\right)$, $39.9\left(\mathrm{CH}_{2}\right), 37.6\left(\mathrm{CH}_{2}\right), 24.6\left(\mathrm{CH}_{2}\right), 14.5\left(\mathrm{CH}_{3}\right) . \mathrm{MS}, \mathrm{m} / \mathrm{z}$ (\% relative intensity) $296\left(\mathrm{M}^{+}+1,100\right)$, 264 (23), 236 (22). HRMS: calcd.for $\mathrm{C}_{14} \mathrm{H}_{18} \mathrm{NO}_{4} \mathrm{~S}$ : 296.095655; found: 296.096117; calcd. for $\mathrm{C}_{14} \mathrm{H}_{17} \mathrm{NO}_{4} \mathrm{~S}: 295.087830$; found: 295.088507. 
3-Chloromethyl-5,7-dihydro-[2]pyridine-6,6-dicarboxylic acid dimethyl ester (4i)

${ }^{1} \mathrm{H}-\mathrm{NMR}\left(250 \mathrm{MHz}, \mathrm{CDCl}_{3}\right) \delta(\mathrm{ppm}): 8.35(\mathrm{~s}, 1 \mathrm{H}), 7.29(\mathrm{~s}, 1 \mathrm{H}), 4.58(\mathrm{~s}, 2 \mathrm{H}), 3.70(\mathrm{~s}, 6 \mathrm{H}), 3.57(\mathrm{~s}$, 2H), $3.56(\mathrm{~s}, 2 \mathrm{H}) .{ }^{13} \mathrm{C}-\mathrm{NMR}$, DEPT $\left(63 \mathrm{MHz}, \mathrm{CDCl}_{3}\right) \delta(\mathrm{ppm}): 171.1(2 \mathrm{x} \mathrm{C}), 154.9(\mathrm{C}), 150.7(\mathrm{C})$, $144.9(\mathrm{CH}), 135.6(\mathrm{C}), 118.7(\mathrm{CH}), 60.0(\mathrm{C}), 53.1\left(2 \times \mathrm{CH}_{3}\right), 46.6\left(\mathrm{CH}_{2}\right), 40.1\left(\mathrm{CH}_{2}\right), 37.9\left(\mathrm{CH}_{2}\right)$. MS, m/z (\% relative intensity) $286\left(\mathrm{M}^{+}+3,24\right), 284\left(\mathrm{M}^{+}+1,98\right), 248$ (100). HRMS: calcd. for $\mathrm{C}_{13} \mathrm{H}_{15} \mathrm{NO}_{4}{ }^{37} \mathrm{Cl}$ : 286.066011; found: 286.066188; calcd. for $\mathrm{C}_{13} \mathrm{H}_{15} \mathrm{NO}_{4}{ }^{35} \mathrm{Cl}$ : 284.068961 ; found: 284.068930; calcd. for $\mathrm{C}_{13} \mathrm{H}_{14} \mathrm{NO}_{4}{ }^{35} \mathrm{Cl}$ : 283.061136; found: 283.061284 .

\section{3-Cyanomethyl-5,7-dihydro-[2]pyridine-6,6-dicarboxylic acid dimethyl ester (4r)}

${ }^{1} \mathrm{H}-\mathrm{NMR}\left(250 \mathrm{MHz}, \mathrm{CDCl}_{3}\right) \delta(\mathrm{ppm}): 8.31(\mathrm{~s}, 1 \mathrm{H}), 7.22(\mathrm{~s}, 1 \mathrm{H}), 3.82(\mathrm{~s}, 2 \mathrm{H}), 3.68(\mathrm{~s}, 6 \mathrm{H}), 3.53(\mathrm{~s}$, 2H), $3.52(\mathrm{~s}, 2 \mathrm{H}) .{ }^{13} \mathrm{C}-\mathrm{NMR}$, DEPT $\left(63 \mathrm{MHz}, \mathrm{CDCl}_{3}\right) \delta(\mathrm{ppm}): 170.9(2 \mathrm{x} \mathrm{C}), 151,1(\mathrm{C}), 148,6(\mathrm{C})$, $145.2(\mathrm{CH}), 135.5(\mathrm{C}), 118.1(\mathrm{CH}), 117.0(\mathrm{C}), 59.8(\mathrm{C}), 53.0\left(2 \mathrm{x} \mathrm{CH}_{3}\right), 39.9\left(\mathrm{CH}_{2}\right), 37,6\left(\mathrm{CH}_{2}\right)$, $26.0\left(\mathrm{CH}_{2}\right) . \mathrm{MS}, \mathrm{m} / \mathrm{z}$ (\% relative intensity) $275\left(\mathrm{M}^{+}+1,100\right), 243(13), 215$ (11). HRMS: calcd.for $\mathrm{C}_{14} \mathrm{H}_{15} \mathrm{~N}_{2} \mathrm{O}_{4}$ : 275.103182; found: 275.102322; calcd. for $\mathrm{C}_{14} \mathrm{H}_{14} \mathrm{~N}_{2} \mathrm{O}_{4}$ : 274.095357; found: 274.095857.

3-(Cyanodimethylmethyl)-5,7-dihydro-[2]pyridine-6,6-dicarboxylic acid dimethyl ester (4r') ${ }^{1} \mathrm{H}-\mathrm{NMR}\left(250 \mathrm{MHz}, \mathrm{CDCl}_{3}\right) \delta(\mathrm{ppm}): 8.41(\mathrm{~s}, 1 \mathrm{H}), 7.40(\mathrm{~s}, 1 \mathrm{H}), 3.70(\mathrm{~s}, 6 \mathrm{H}), 3.55(\mathrm{~s}, 4 \mathrm{H}), 1.67$ (s, 6H). ${ }^{13} \mathrm{C}-\mathrm{NMR}$, DEPT $\left(63 \mathrm{MHz}, \mathrm{CDCl}_{3}\right) \delta(\mathrm{ppm}): 171.1(2 \times \mathrm{C}), 157.9(\mathrm{C}), 150.8(\mathrm{C}), 144.9(\mathrm{C})$, $135.1(\mathrm{C}), 124.2(\mathrm{C}), 115.6(\mathrm{CH}), 59.9(\mathrm{C}), 53.0\left(2 \times \mathrm{CH}_{3}\right), 40.1\left(\mathrm{CH}_{2}\right), 39.2(\mathrm{C}), 37.8\left(\mathrm{CH}_{2}\right), 27.7$ $\left(2 \times \mathrm{CH}_{3}\right) . \mathrm{MS}, \mathrm{m} / \mathrm{z}$ (\% relative intensity) $303\left(\mathrm{M}^{+}+1,100\right), 276$ (76), 243 (42). HRMS: calcd. for $\mathrm{C}_{16} \mathrm{H}_{19} \mathrm{~N}_{2} \mathrm{O}_{4}$ : 303.134482; found: 303.133521 .

3-(2-Cyanovinyl)-5,7-dihydro-[2]pyridine-6,6-dicarboxylic acid dimethyl ester (4s)

${ }^{1} \mathrm{H}-\mathrm{NMR}\left(250 \mathrm{MHz}, \mathrm{CDCl}_{3}\right) \delta(\mathrm{ppm}): 8.43(\mathrm{~s}, 1 \mathrm{H}), 7.32(\mathrm{~d}, \mathrm{~J}=16.1 \mathrm{~Hz}, 1 \mathrm{H}), 7.17(\mathrm{~s}, 1 \mathrm{H}), 6.48(\mathrm{~d}$, $\mathrm{J}=16.1 \mathrm{~Hz}, 1 \mathrm{H}), 3.74(\mathrm{~s}, 6 \mathrm{H}), 3.62(\mathrm{~s}, 2 \mathrm{H}), 3.58(\mathrm{~s}, 2 \mathrm{H}) .{ }^{13} \mathrm{C}-\mathrm{NMR}$, DEPT $\left(63 \mathrm{MHz}, \mathrm{CDCl}_{3}\right) \delta$ (ppm): 171 (2 x C), $150.6(\mathrm{C}), 149.9(\mathrm{C}), 148.7(\mathrm{CH}), 146.0(\mathrm{CH}), 138.1(\mathrm{C}), 120.1(\mathrm{CH}), 118.0$ (C), $100.1(\mathrm{CH}), 59.9(\mathrm{C}), 53.2\left(2 \mathrm{x} \mathrm{CH}_{3}\right), 40.0\left(\mathrm{CH}_{2}\right), 38.2\left(\mathrm{CH}_{2}\right) . \mathrm{MS}, \mathrm{m} / \mathrm{z}$ (\% relative intensity) $287\left(\mathrm{M}^{+}+1\right.$, 100). HRMS: calcd.for $\mathrm{C}_{15} \mathrm{H}_{15} \mathrm{~N}_{2} \mathrm{O}_{4}$ : 287.103182; found: 287.103357 ; calcd. for $\mathrm{C}_{15} \mathrm{H}_{14} \mathrm{~N}_{2} \mathrm{O}_{4}$ : 286.095357; found: 286.094273 .

\section{3-(2-Cyanophenyl)-5,7-dihydro-[2]pyridine-6,6-dicarboxylic acid dimethyl ester (4t)}

${ }^{1} \mathrm{H}-\mathrm{NMR}\left(250 \mathrm{MHz}, \mathrm{CDCl}_{3}\right) \delta(\mathrm{ppm}): 8.58(\mathrm{~s}, 1 \mathrm{H}), 7.76(\mathrm{~d}, \mathrm{~J}=7.6 \mathrm{~Hz}, 2 \mathrm{H}), 7.64(\mathrm{t}, \mathrm{J}=7.6 \mathrm{~Hz}, 1 \mathrm{H})$, $7.59(\mathrm{~s}, 1 \mathrm{H}), 7.46(\mathrm{t} \mathrm{J}=7.6 \mathrm{~Hz}, 1 \mathrm{H}), 3.76(\mathrm{~s}, 6 \mathrm{H}), 3.68(\mathrm{~s}, 4 \mathrm{H}) .{ }^{13} \mathrm{C}-\mathrm{NMR}$, DEPT $\left(63 \mathrm{MHz}, \mathrm{CDCl}_{3}\right) \delta$ (ppm): $171.2(2 \times \mathrm{C}), 153.8(\mathrm{C}), 150.5(\mathrm{C}), 145.4(\mathrm{CH}), 143.4(\mathrm{C}), 136.0(\mathrm{C}), 134.0(\mathrm{CH}), 133.4$ $(\mathrm{CH}), 133.0(\mathrm{CH}), 132.6(\mathrm{CH}), 118.9(\mathrm{CH}) 110.8(\mathrm{C}), 59.9(\mathrm{C}), 53.1\left(2 \mathrm{x} \mathrm{CH}_{3}\right), 40.2\left(\mathrm{CH}_{2}\right), 38.0$ $\left(\mathrm{CH}_{2}\right)$. MS, $\mathrm{m} / \mathrm{z}$ (\% relative intensity) $337\left(\mathrm{M}^{+}+1\right.$, 100). HRMS: calcd.for $\mathrm{C}_{19} \mathrm{H}_{17} \mathrm{~N}_{2} \mathrm{O}_{4}$ : 337.118832; found: 337.118282 .

3-(4-Cyanophenyl)-5,7-dihydro-[2]pyridine-6,6-dicarboxylic acid dimethyl ester (4u)

${ }^{1} \mathrm{H}-\mathrm{NMR}\left(250 \mathrm{MHz}, \mathrm{CDCl}_{3}\right) \delta(\mathrm{ppm}): 8.53(\mathrm{~s}, 1 \mathrm{H}), 8.03(\mathrm{~d}, \mathrm{~J}=8.4 \mathrm{~Hz}, 2 \mathrm{H}), 7.70(\mathrm{~d}, \mathrm{~J}=8.4 \mathrm{~Hz}, 2 \mathrm{H})$, $7.60(\mathrm{~s}, 1 \mathrm{H}), 3.76(\mathrm{~s}, 6 \mathrm{H}), 3.66(\mathrm{~s}, 2 \mathrm{H}) 3.85(\mathrm{~s}, 2 \mathrm{H}) .{ }^{13} \mathrm{C}-\mathrm{NMR}, \mathrm{DEPT}\left(63 \mathrm{MHz}, \mathrm{CDCl}_{3}\right) \delta(\mathrm{ppm})$ : $171.2(2 \times \mathrm{C}), 153.9(\mathrm{C}), 150.8(\mathrm{C}), 145.6(\mathrm{CH}), 143.4(\mathrm{C}), 136.0(\mathrm{C}), 132.4(2 \times \mathrm{CH}), 127.3(2 \times$ $\mathrm{CH}), 118.7(\mathrm{C}), 116.8(\mathrm{CH}), 112.0(\mathrm{C}), 60.0(\mathrm{C}), 53.2\left(2 \times \mathrm{CH}_{3}\right), 40.2\left(\mathrm{CH}_{2}\right), 38.0\left(\mathrm{CH}_{2}\right) . \mathrm{MS}, \mathrm{m} / \mathrm{z}$ (\% relative intensity) $337\left(\mathrm{M}^{+}+1,100\right)$. HRMS: calcd.for $\mathrm{C}_{19} \mathrm{H}_{17} \mathrm{~N}_{2} \mathrm{O}_{4}$ : 337.118832; found: 337.117530 .

3-(6-Cyanopyridin-2-yl)-5,7-dihydro-[2]pyridine-6,6-dicarboxylic acid dimethyl ester (4v) ${ }^{1} \mathrm{H}-\mathrm{NMR}\left(250 \mathrm{MHz}, \mathrm{CDCl}_{3}\right) \delta(\mathrm{ppm}): 8.56(\mathrm{~d}, \mathrm{~J}=8.3 \mathrm{~Hz}, 1 \mathrm{H}), 8.45(\mathrm{~s}, 1 \mathrm{H}), 8,26(\mathrm{~s}, 1 \mathrm{H}), 7.9(\mathrm{t}, \mathrm{J}=$ $8.3 \mathrm{~Hz}, 1 \mathrm{H}), 7.65(\mathrm{~d}, \mathrm{~J}=8.3 \mathrm{~Hz}, 1 \mathrm{H}), 3.73(\mathrm{~s}, 3 \mathrm{H}), 3.65(\mathrm{~s}, 4 \mathrm{H}) .{ }^{13} \mathrm{C}-\mathrm{NMR}$, DEPT $\left(63 \mathrm{MHz}, \mathrm{CDCl}_{3}\right)$ $\delta$ (ppm): 171.2 (2 x C), $157.5(\mathrm{C}), 152.7(\mathrm{C}), 150.9(\mathrm{C}), 144.8(\mathrm{CH}), 137.7(\mathrm{CH}), 137.5(\mathrm{C}), 132.9$ 
(C), $127.9(\mathrm{CH}), 124.2(\mathrm{CH}), 117.4(\mathrm{CH}), 117.3(\mathrm{C}), 60.0(\mathrm{C}), 53.15\left(2 \times \mathrm{CH}_{3}\right), 40.2\left(\mathrm{CH}_{2}\right), 38.12$ $\left(\mathrm{CH}_{2}\right) . \mathrm{MS}, \mathrm{m} / \mathrm{z}$ (\% relative intensity) $338\left(\mathrm{M}^{+}+1,100\right), 306$ (6), 278 (6). HRMS: calcd.for $\mathrm{C}_{18} \mathrm{H}_{16} \mathrm{~N}_{3} \mathrm{O}_{4}$ : 338.114081; found: 338.115307.

6-Cyano-1,3,4,5-tetrahydroindene-2,2-dicarboxylic acid dimethyl ester (5)

${ }^{1} \mathrm{H}-\mathrm{NMR}\left(250 \mathrm{MHz}, \mathrm{CDCl}_{3}\right) \delta(\mathrm{ppm}): 6.60(\mathrm{~s}, 1 \mathrm{H}), 3.74(\mathrm{~s}, 6 \mathrm{H}), 3.09(\mathrm{~s}, 4 \mathrm{H}), 2.46(\mathrm{t}, \mathrm{J}=9.8 \mathrm{~Hz}$, 2H), $2.29(\mathrm{t}, \mathrm{J}=9.8 \mathrm{~Hz}, 2 \mathrm{H}) .{ }^{13} \mathrm{C}-\mathrm{NMR}$, DEPT $\left(63 \mathrm{MHz}, \mathrm{CDCl}_{3}\right) \delta(\mathrm{ppm}): 170.9(2 \times \mathrm{C}), 139.7(\mathrm{C})$, $135.6(\mathrm{CH}), 128.5(\mathrm{C}), 105.6(\mathrm{C}), 57.1(\mathrm{C}), 52.1\left(2 \times \mathrm{CH}_{3}\right), 42.5\left(\mathrm{CH}_{2}\right), 39.6\left(\mathrm{CH}_{2}\right), 23.4\left(\mathrm{CH}_{2}\right)$, $21.7\left(\mathrm{CH}_{2}\right) . \mathrm{MS}, \mathrm{m} / \mathrm{z}$ (\% relative intensity) $262\left(\mathrm{M}^{+}+1,100\right), 202(17)$.

Pyridine-2,3,6-tricarboxylic acid trimethyl ester (13ax)

${ }^{1} \mathrm{H}-\mathrm{NMR}\left(250 \mathrm{MHz}, \mathrm{CDCl}_{3}\right) \delta(\mathrm{ppm}): 8.28(\mathrm{~d}, \mathrm{~J}=8.1 \mathrm{~Hz}, 1 \mathrm{H}), 8.22(\mathrm{~d}, \mathrm{~J}=8.1 \mathrm{~Hz}, 1 \mathrm{H}), 3.96(\mathrm{~s}, 3 \mathrm{H})$, $3.94(\mathrm{~s}, 3 \mathrm{H}), 3.89$ (s, 3H). ${ }^{13} \mathrm{C}-\mathrm{NMR}$, DEPT $\left(63 \mathrm{MHz}, \mathrm{CDCl}_{3}\right) \delta(\mathrm{ppm}): 165.7(\mathrm{C}), 164.8(\mathrm{C}), 164.1$ (C), $150.8(\mathrm{C}), 149.5(\mathrm{C}), 138.9(\mathrm{CH}), 128.6(\mathrm{C}), 125.9(\mathrm{C}), 53.2\left(3 \times \mathrm{CH}_{3}\right) . \mathrm{MS}, \mathrm{m} / \mathrm{z}$ (\% relative intensity) $254\left(\mathrm{M}^{+}+1,100\right), 222$ (26). HRMS: calcd.for $\mathrm{C}_{11} \mathrm{H}_{12} \mathrm{NO}_{6}$ : 254.066462; found: 254.065702 .

6-(Toluene-4-sulfonyl)pyridine-2,5-dicarboxylic acid dimethyl ester (13ay)

${ }^{1} \mathrm{H}-\mathrm{NMR}\left(250 \mathrm{MHz}, \mathrm{CDCl}_{3}\right) \delta(\mathrm{ppm}): 8.20(\mathrm{~d}, \mathrm{~J}=7.5 \mathrm{~Hz}, 1 \mathrm{H}), 8.03(\mathrm{~d}, \mathrm{~J}=7.5 \mathrm{~Hz}, 1 \mathrm{H}), 7.96(\mathrm{~d}, \mathrm{~J}=$ $8.3 \mathrm{~Hz}, 2 \mathrm{H}), 7.31(\mathrm{~d}, \mathrm{~J}=8.3 \mathrm{~Hz}, 2 \mathrm{H}), 4.00(\mathrm{~s}, 3 \mathrm{H}), 3.90(\mathrm{~s}, 3 \mathrm{H}), 2.36(\mathrm{~s}, 3 \mathrm{H}) .{ }^{13} \mathrm{C}-\mathrm{NMR}$, DEPT $(63$ $\left.\mathrm{MHz}, \mathrm{CDCl}_{3}\right) \delta(\mathrm{ppm}): 165.7(\mathrm{C}), 163.3(\mathrm{C}), 155.6(\mathrm{C}), 148.6(\mathrm{C}), 145.2(\mathrm{C}), 138.5(\mathrm{CH}), 135.1$ (C), $131.3(\mathrm{C}), 129.5(\mathrm{CH}), 129.4(\mathrm{CH}), 127.0(\mathrm{CH}), 53.5\left(\mathrm{CH}_{3}\right), 53.0\left(\mathrm{CH}_{3}\right), 21.5\left(\mathrm{CH}_{3}\right) . \mathrm{MS}, \mathrm{m} / \mathrm{z}$ (\% relative intensity) $350\left(\mathrm{M}^{+}+1,100\right), 318$ (11), 196 (16), 125 (43). HRMS: calcd.for $\mathrm{C}_{16} \mathrm{H}_{16} \mathrm{NO}_{6} \mathrm{~S}: 350.069834$; found: 350.070702 .

6-Trichloromethylpyridine-2,5-dicarboxylic acid dimethyl ester (13az)

${ }^{1} \mathrm{H}-\mathrm{NMR}\left(250 \mathrm{MHz}, \mathrm{CDCl}_{3}\right) \delta(\mathrm{ppm}): 8.21(\mathrm{~d}, \mathrm{~J}=7.9 \mathrm{~Hz}, 1 \mathrm{H}), 8.05(\mathrm{~d}, \mathrm{~J}=7.9 \mathrm{~Hz}, 1 \mathrm{H}), 3.99(\mathrm{~s}, 3 \mathrm{H})$, $3.94(\mathrm{~s}, 3 \mathrm{H}) .{ }^{13} \mathrm{C}-\mathrm{NMR}, \mathrm{DEPT}\left(63 \mathrm{MHz}, \mathrm{CDCl}_{3}\right) \delta(\mathrm{ppm}): 166.7(\mathrm{C}), 163.9(\mathrm{C}), 153.9(\mathrm{C}), 147.0$ (C), $139.9(\mathrm{CH}), 129.7(\mathrm{C}), 125.7(\mathrm{CH}), 77.1(\mathrm{C}), 53.2\left(2 \mathrm{x} \mathrm{CH}_{3}\right) . \mathrm{MS}, \mathrm{m} / \mathrm{z}$ (\% relative intensity) $316\left(\mathrm{M}^{+}+5,17\right), 314\left(\mathrm{M}^{+}+3,52\right), 312\left(\mathrm{M}^{+}+1,57\right), 278$ (85), 276 (100), 253 (33), 242 (33), 221 (73). 173 (42). HRMS: calcd. for $\mathrm{C}_{10} \mathrm{H}_{9} \mathrm{Cl}_{3} \mathrm{NO}_{4}$ : 311.959716 ; found: 311.959867 .

3,6-Bistrifluoromethylpyridine-2-carboxylic acid methyl ester (13bx)

${ }^{1} \mathrm{H}-\mathrm{NMR}\left(250 \mathrm{MHz}, \mathrm{CDCl}_{3}\right) \delta(\mathrm{ppm}): 8.23(\mathrm{~d}, \mathrm{~J}=8.3 \mathrm{~Hz}, 1 \mathrm{H}), 7.89(\mathrm{~d}, \mathrm{~J}=8.3 \mathrm{~Hz}, 1 \mathrm{H}), 3.98(\mathrm{~s}, 3 \mathrm{H})$.

\section{References}

(1) Trost, B. M.; Toste, F. D. J. Am. Chem. Soc. 2002, 124, 5025.

(2) Trost, B. M.; Older, C. M. Organometallics 2002, 21, 2544.

(3) Schrenk, J. L.; McNair, A. M.; McCormick, F. B. Mann, K. R. Inorg. Chem. 1986, 25, 3501. 
क्ष

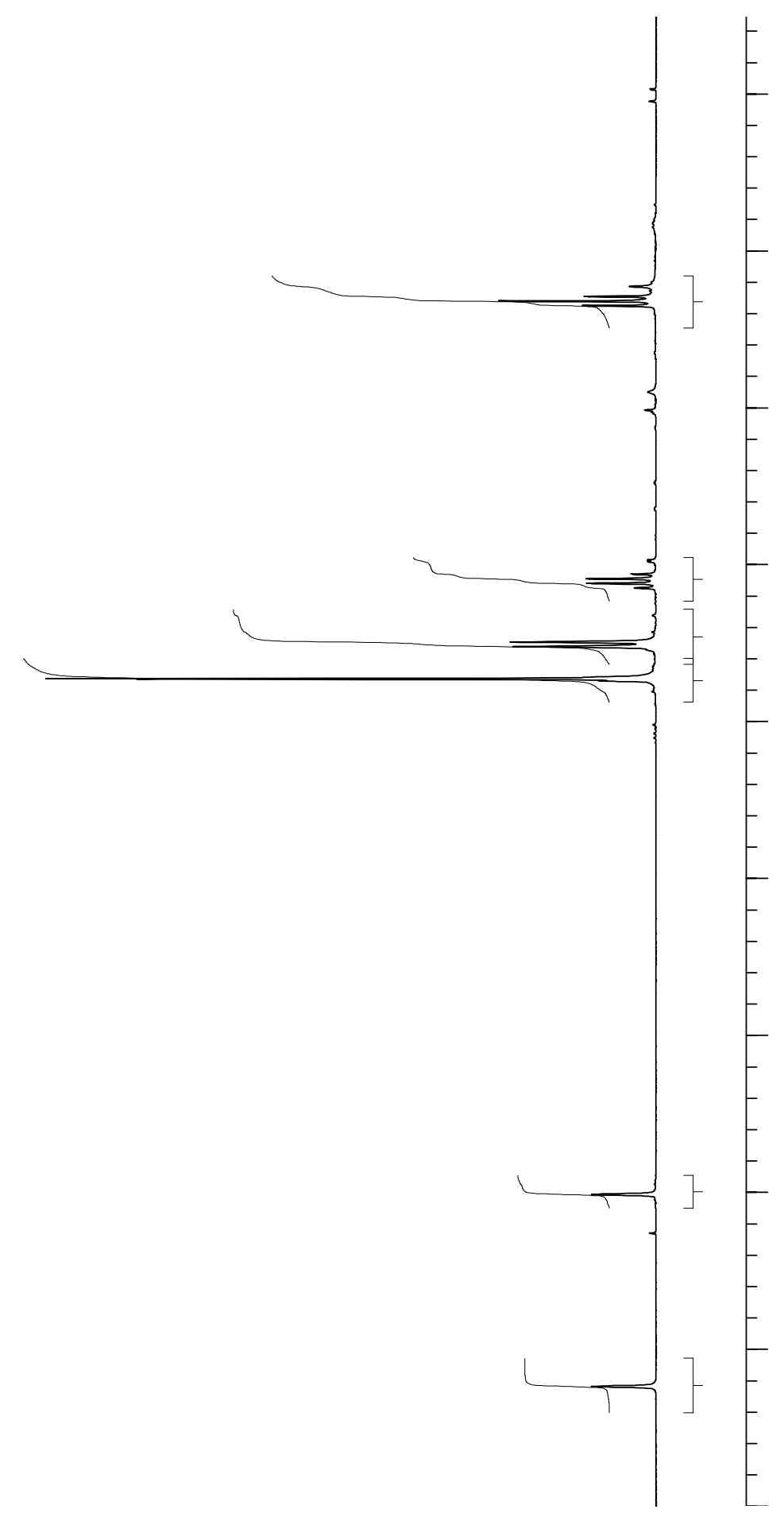



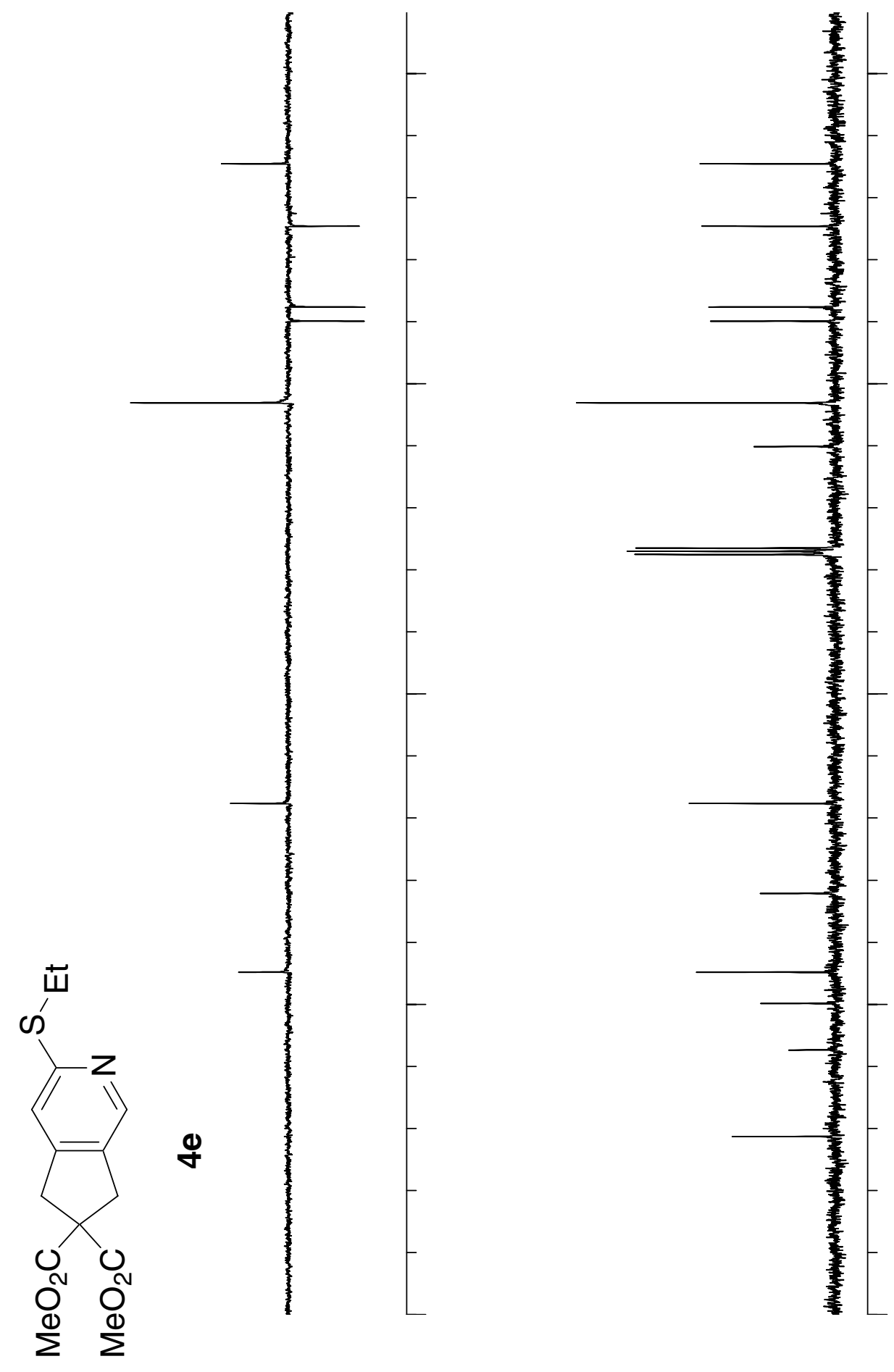


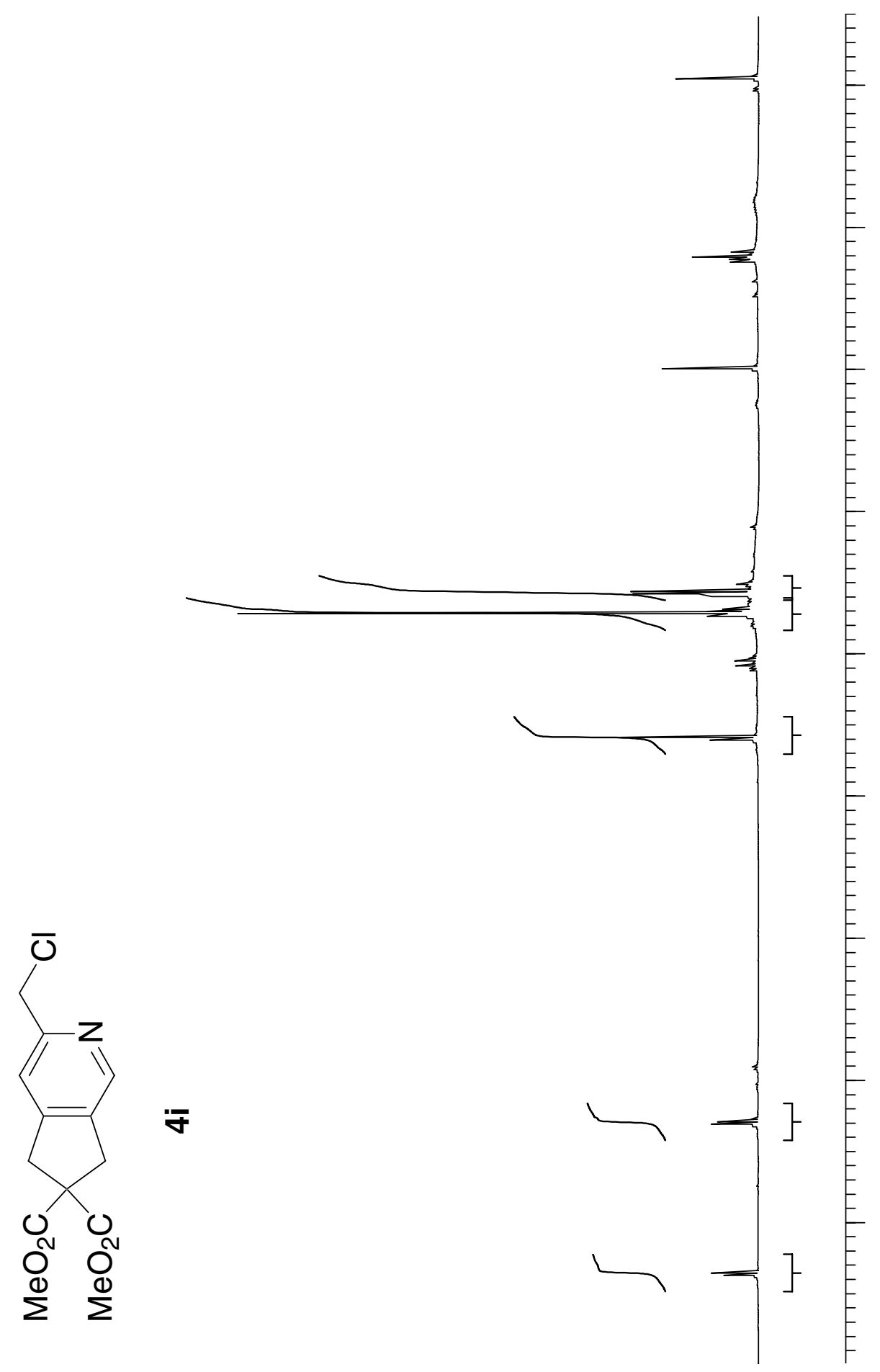



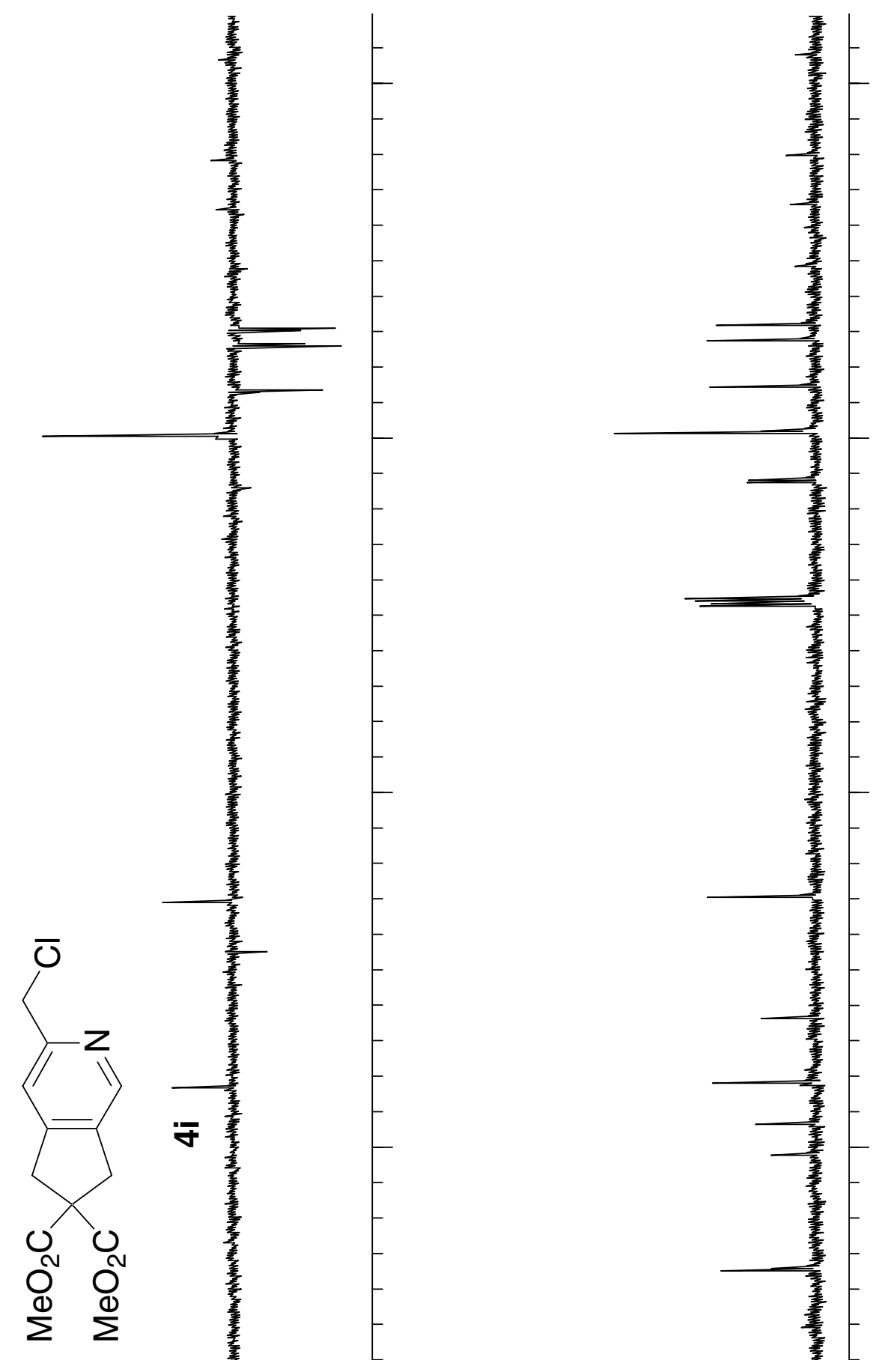


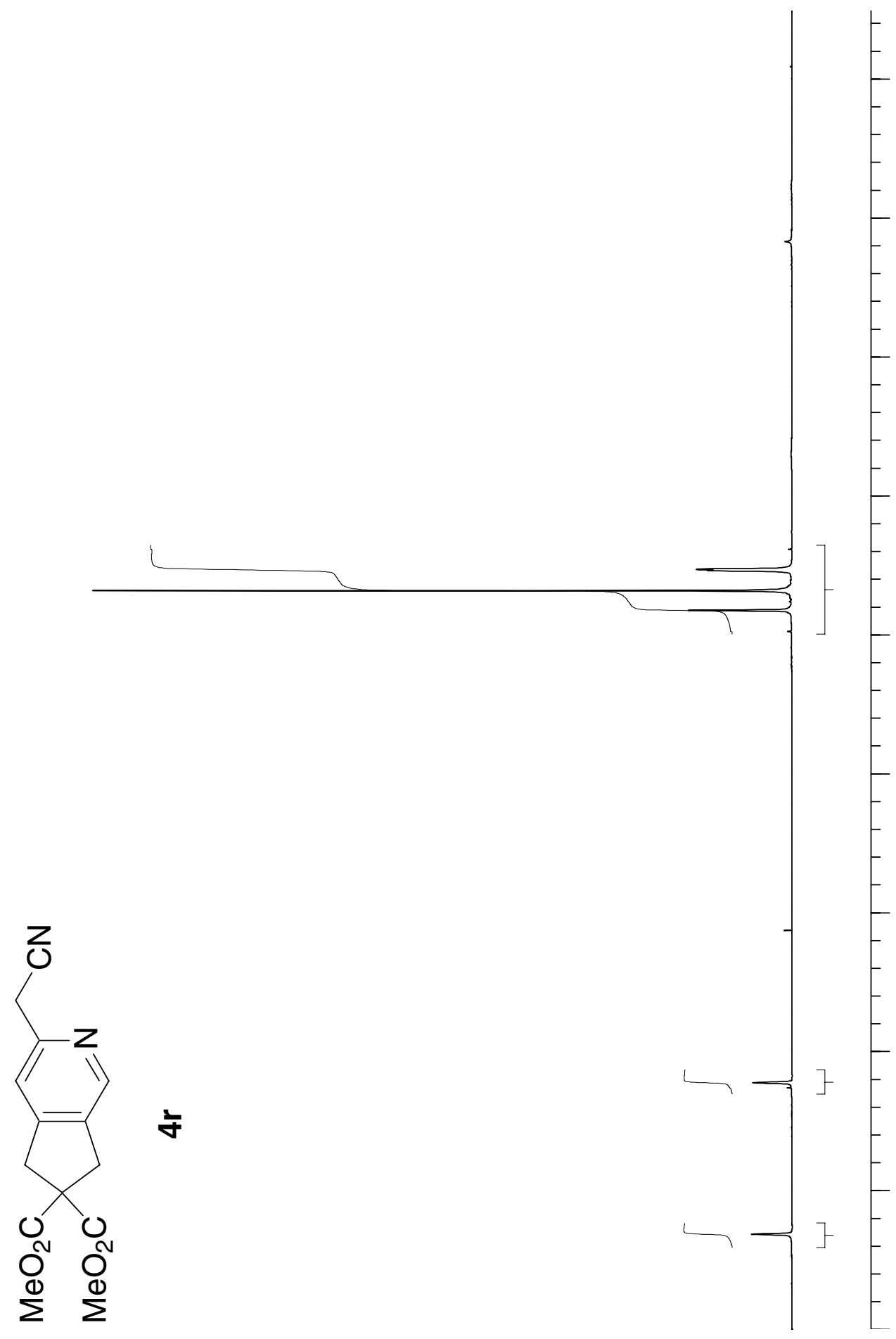


$\frac{\circ}{\bar{n}}$
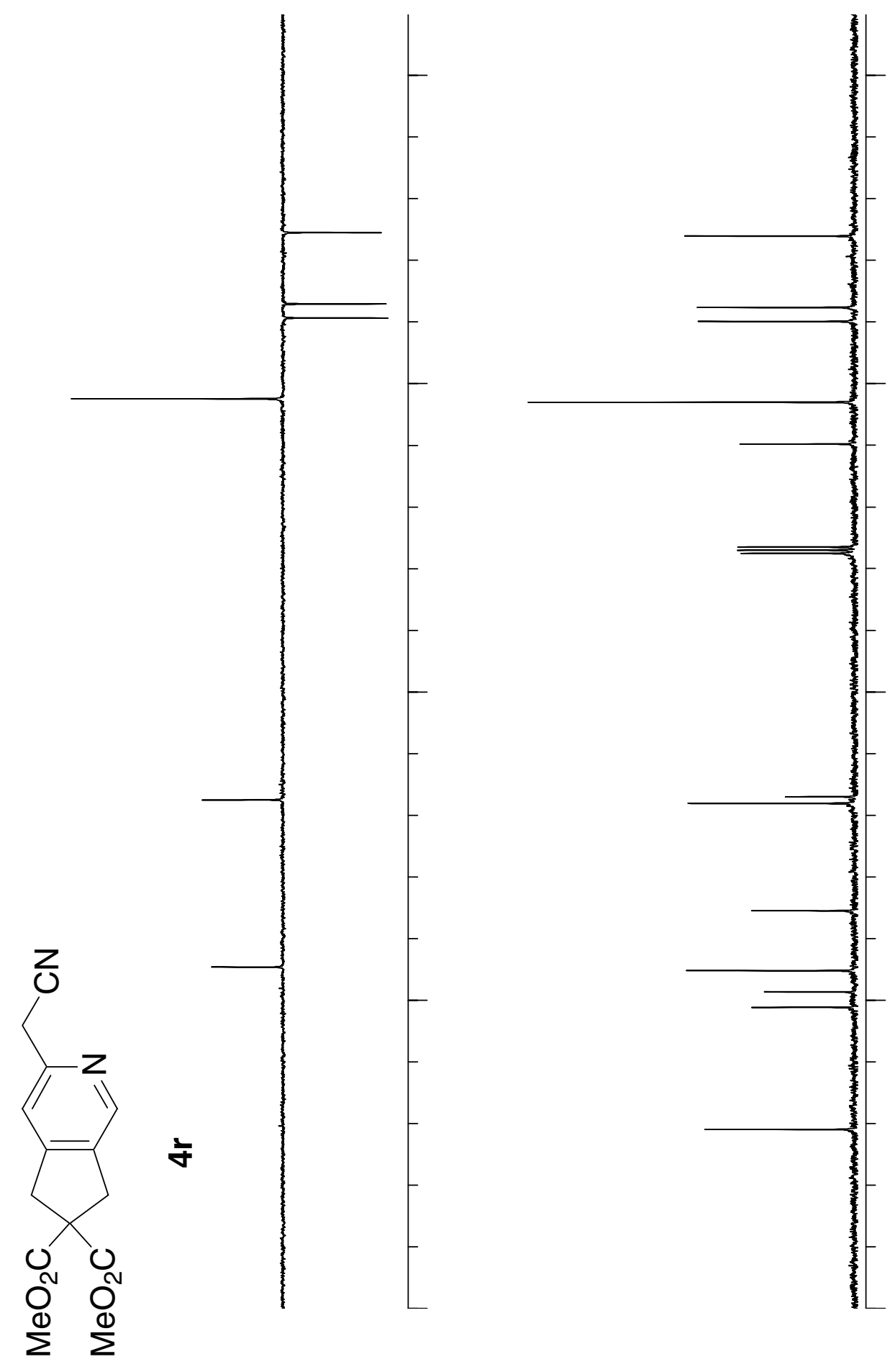
$\overline{5}$

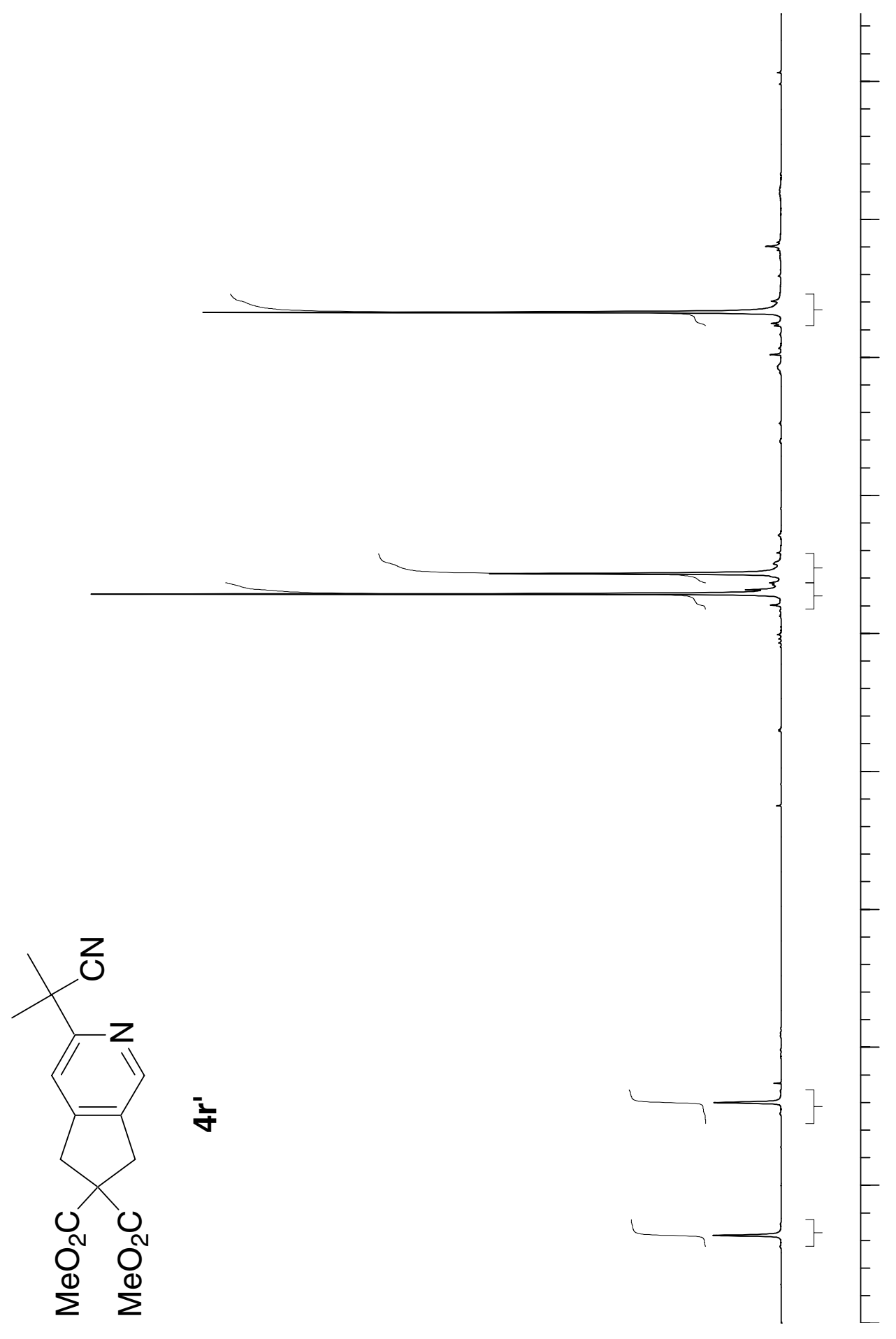


के
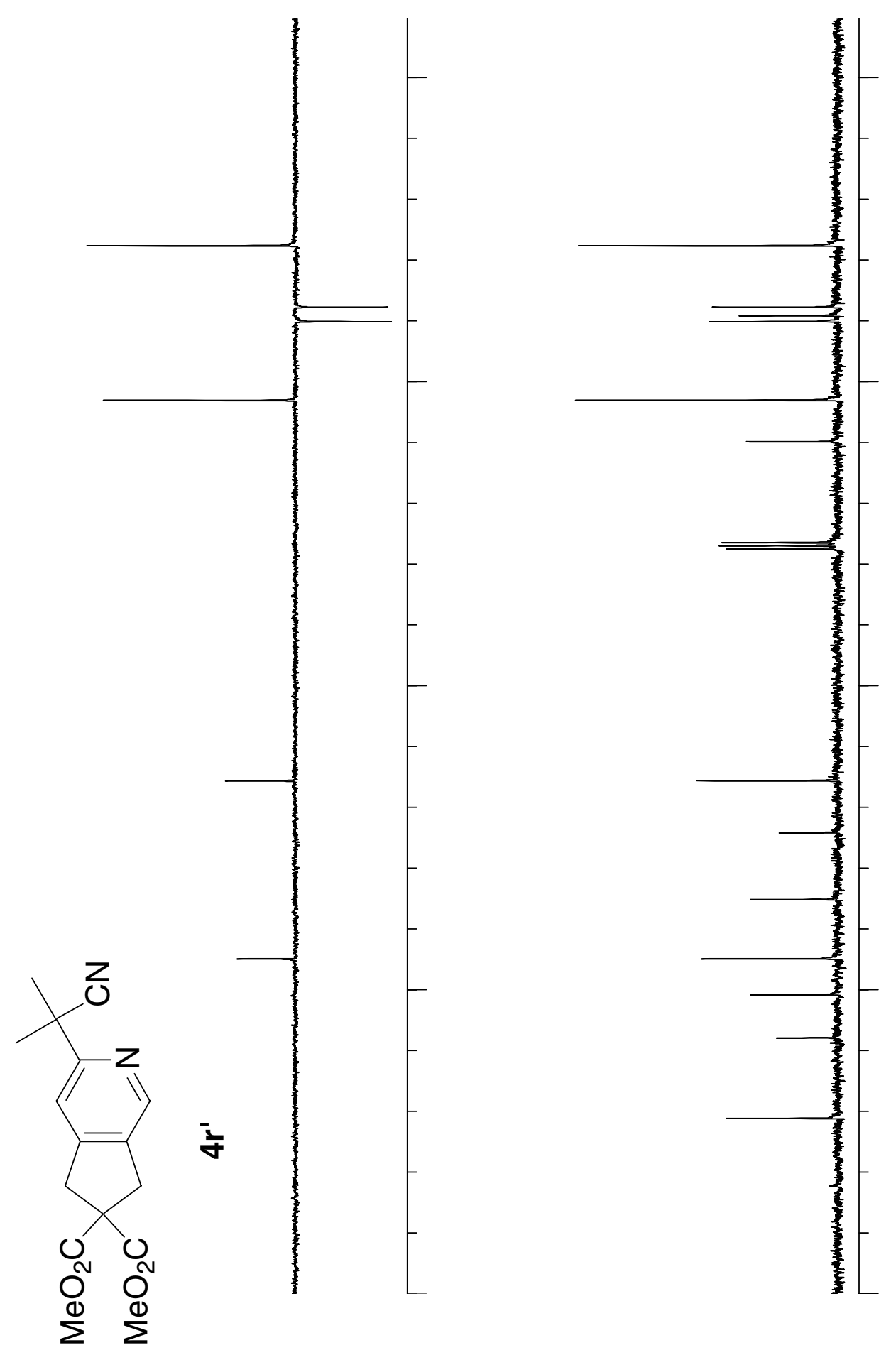
$\frac{m}{2}$

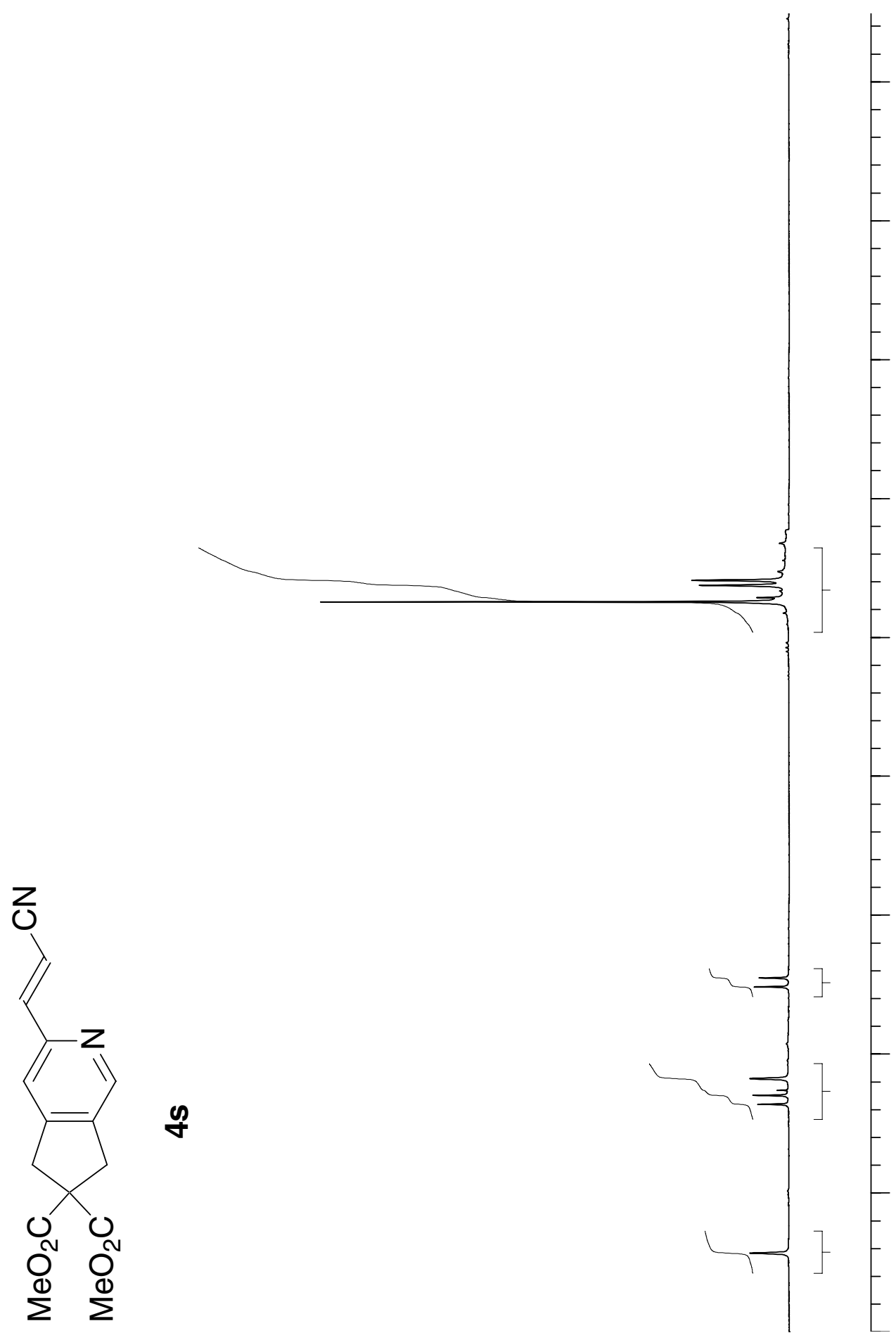


$\pm$
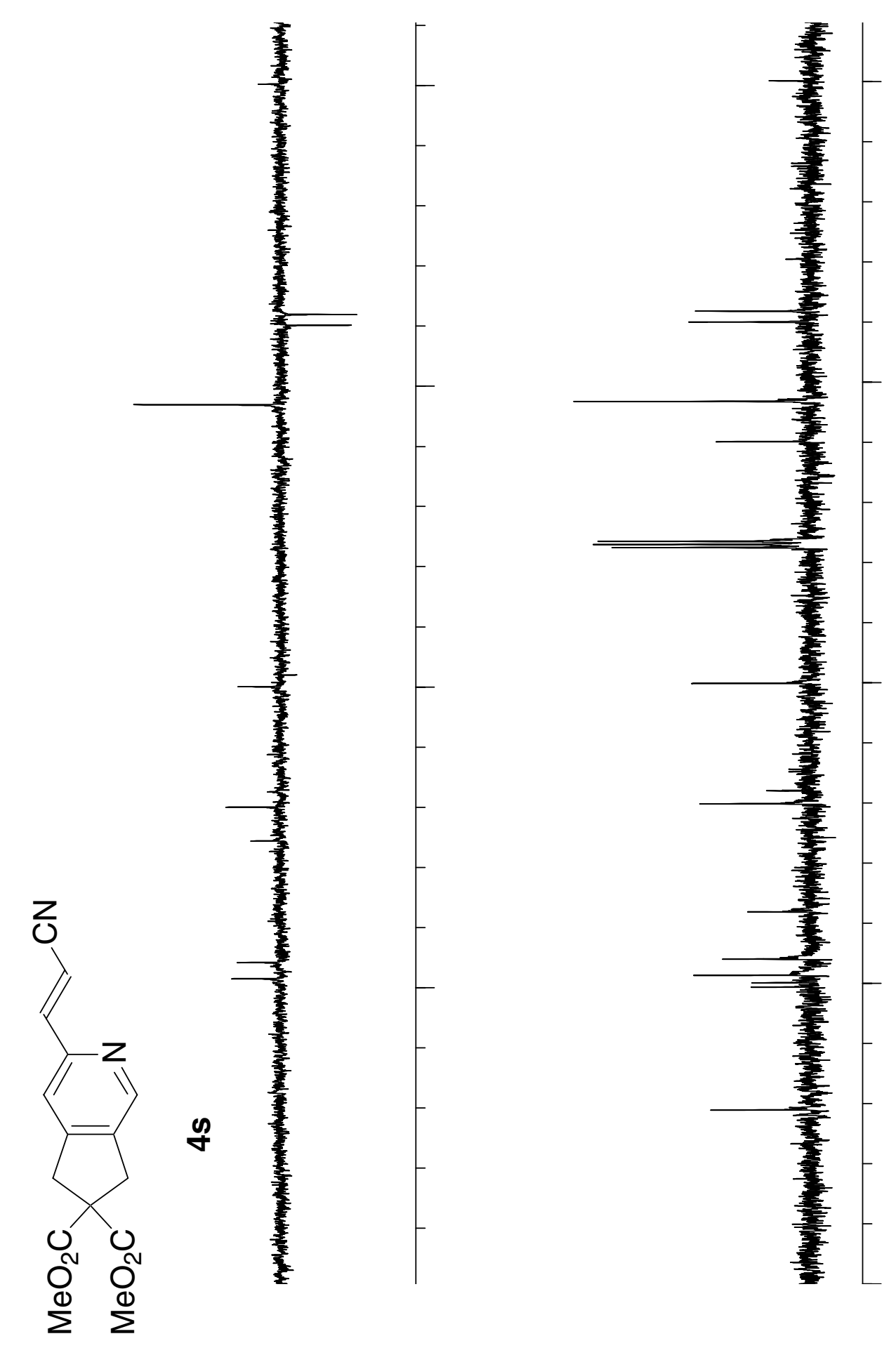
$\frac{n}{n}$

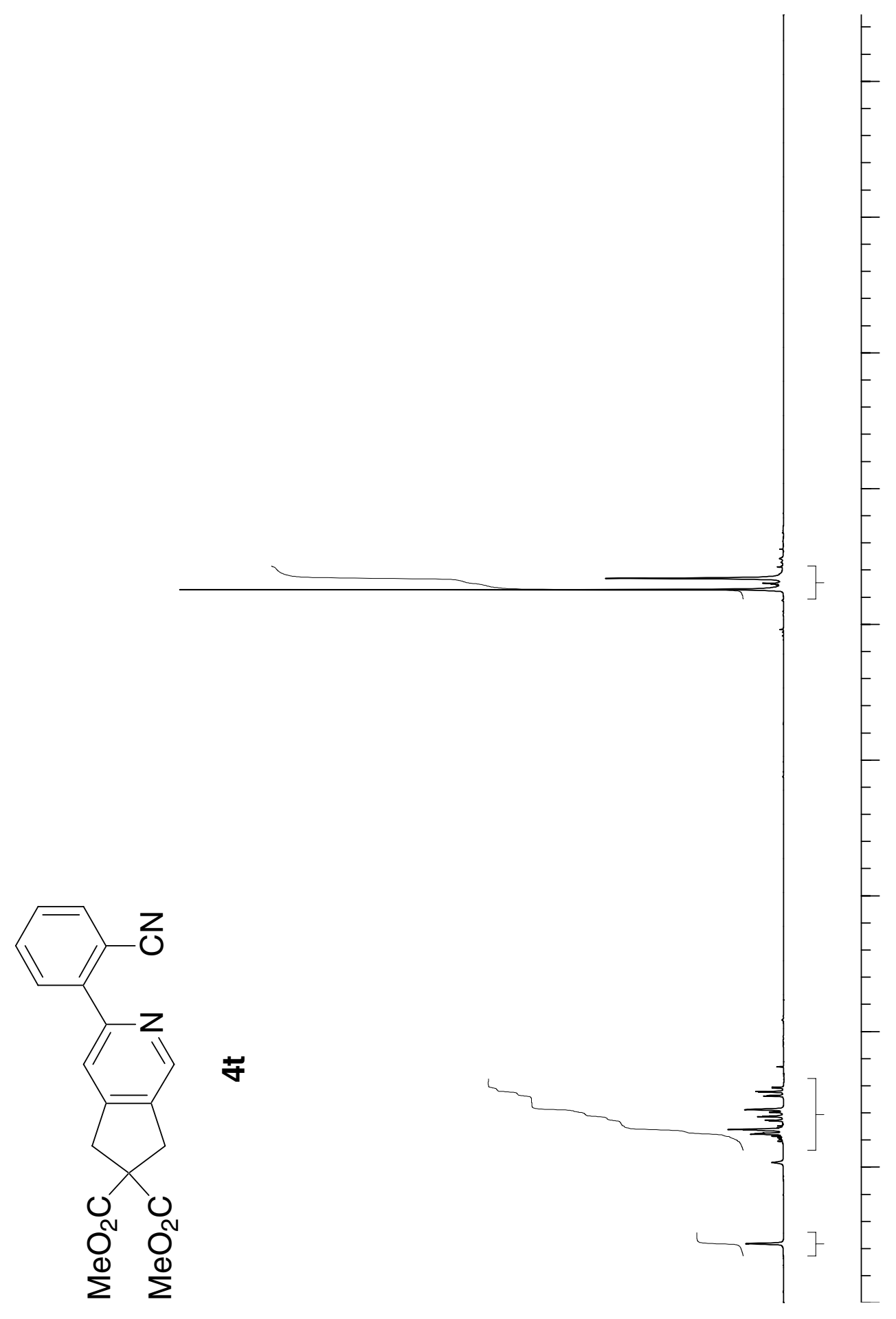


$\frac{\circ}{6}$
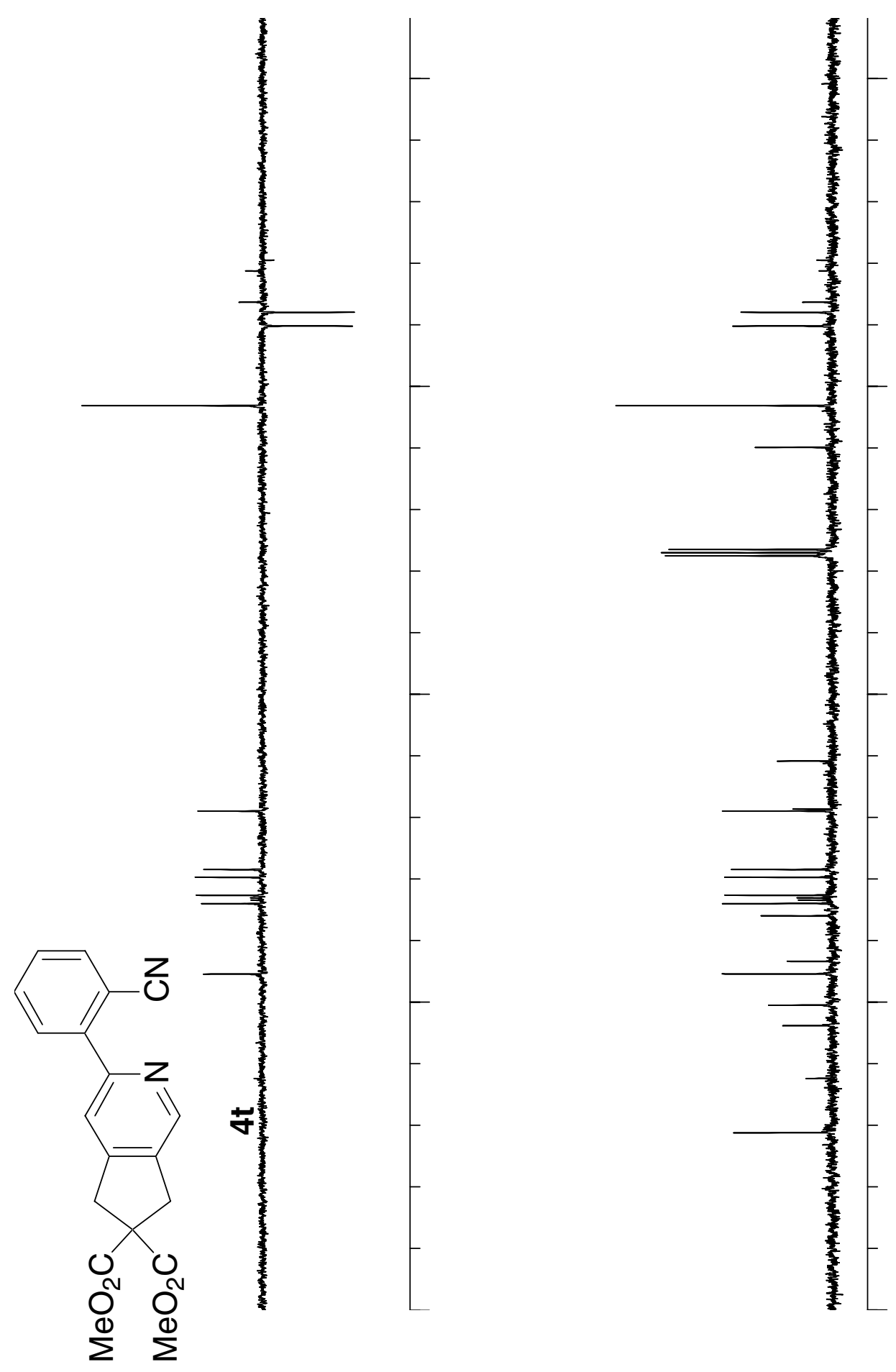
के

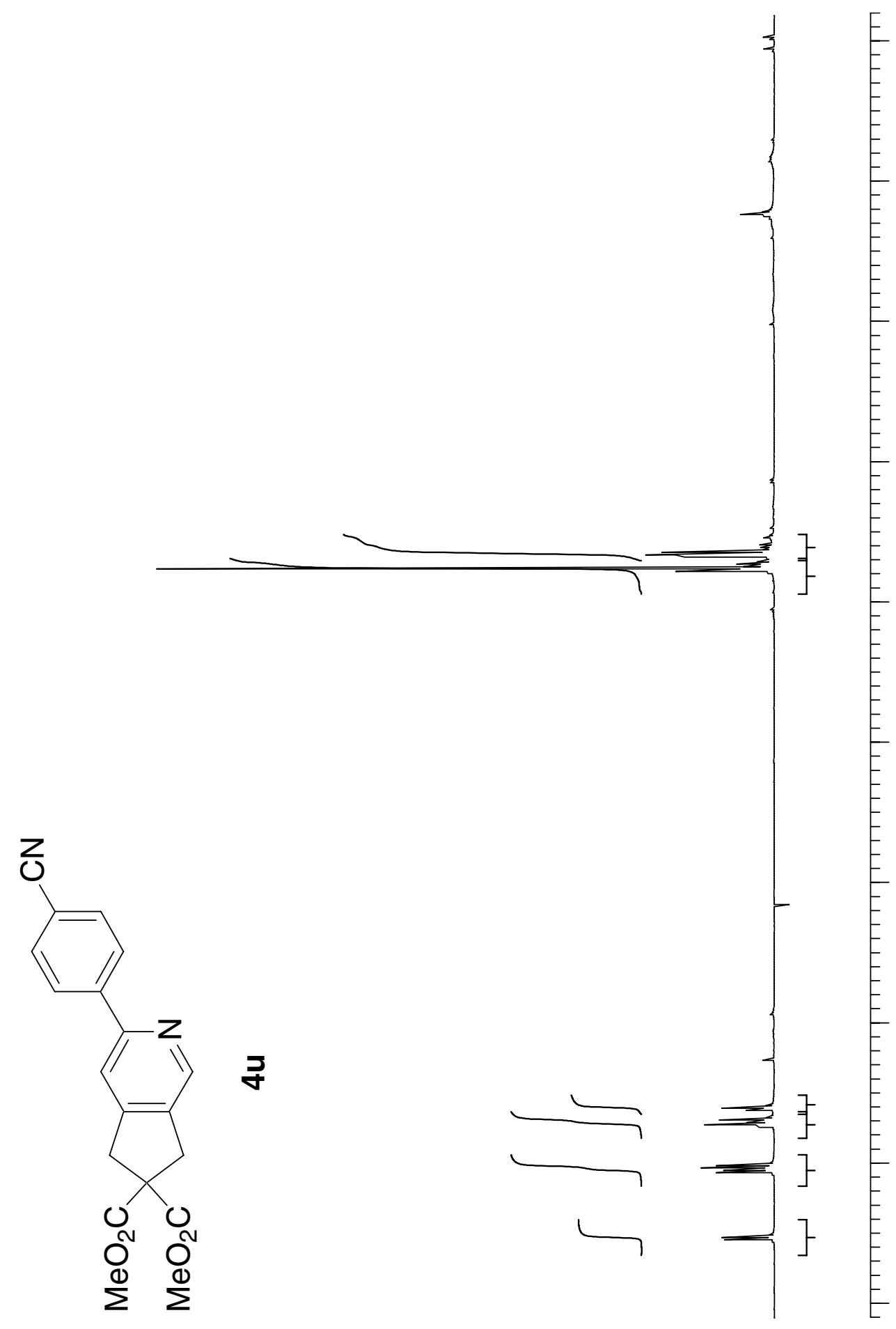


$\infty$
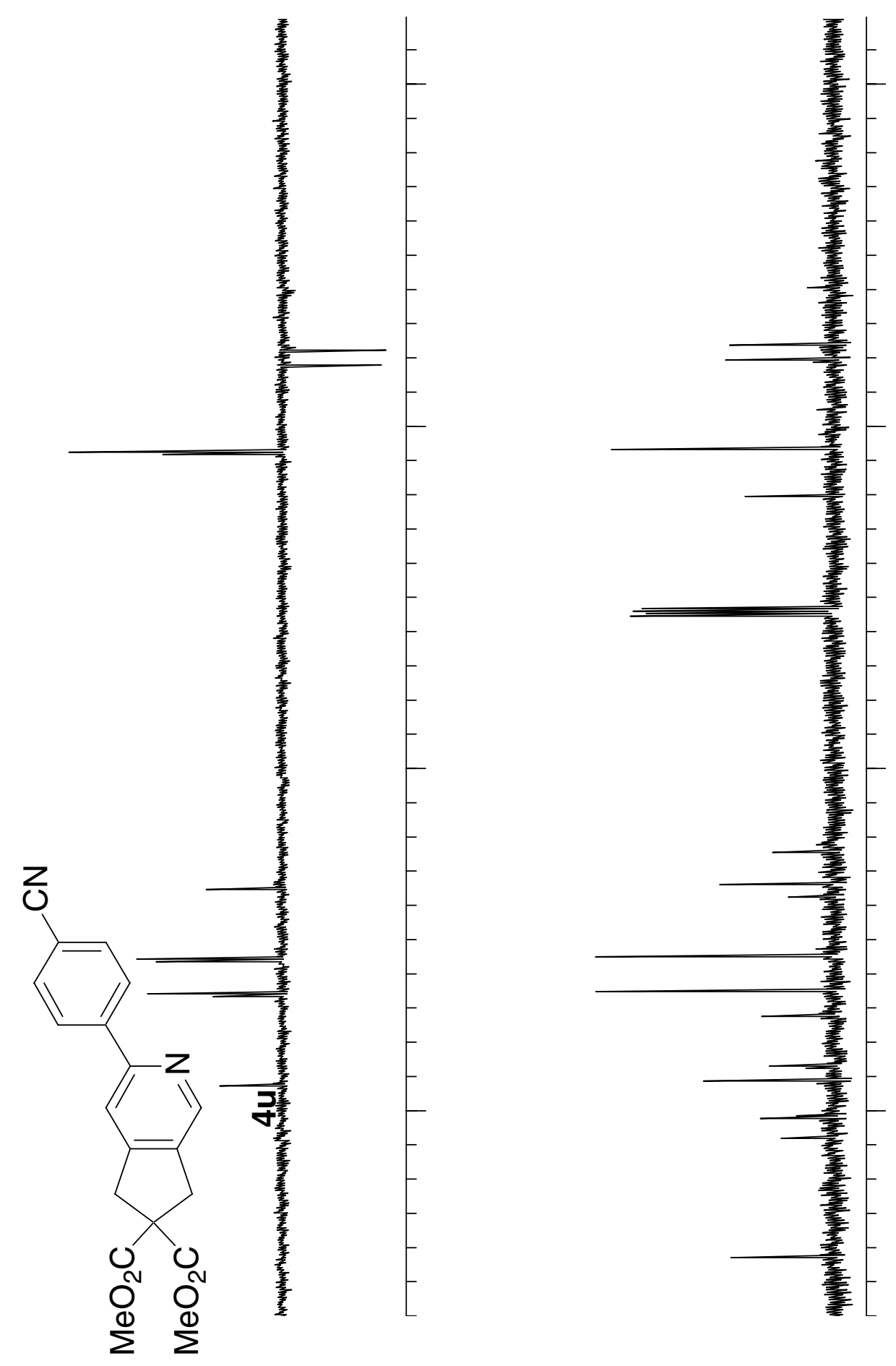
के

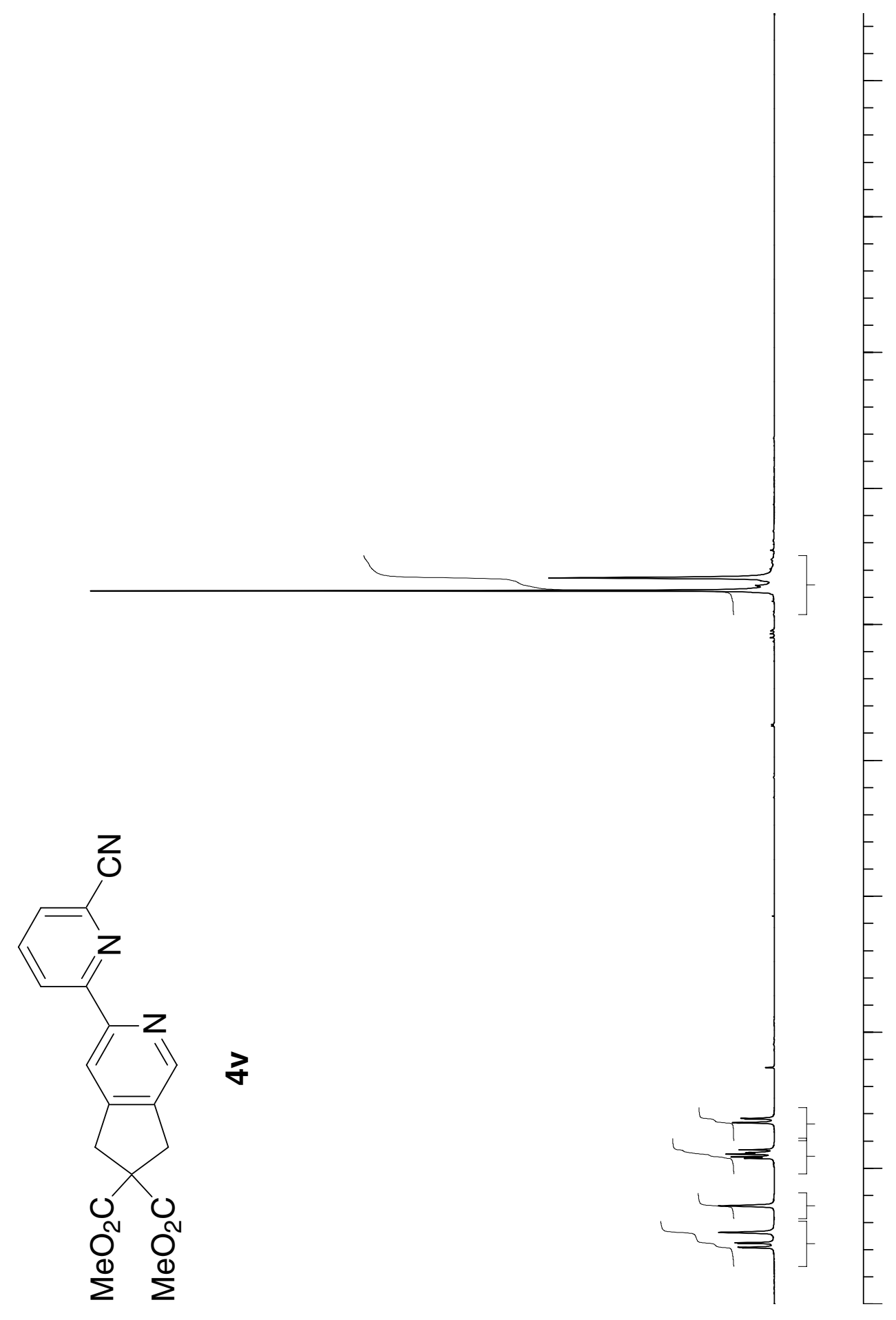


\&
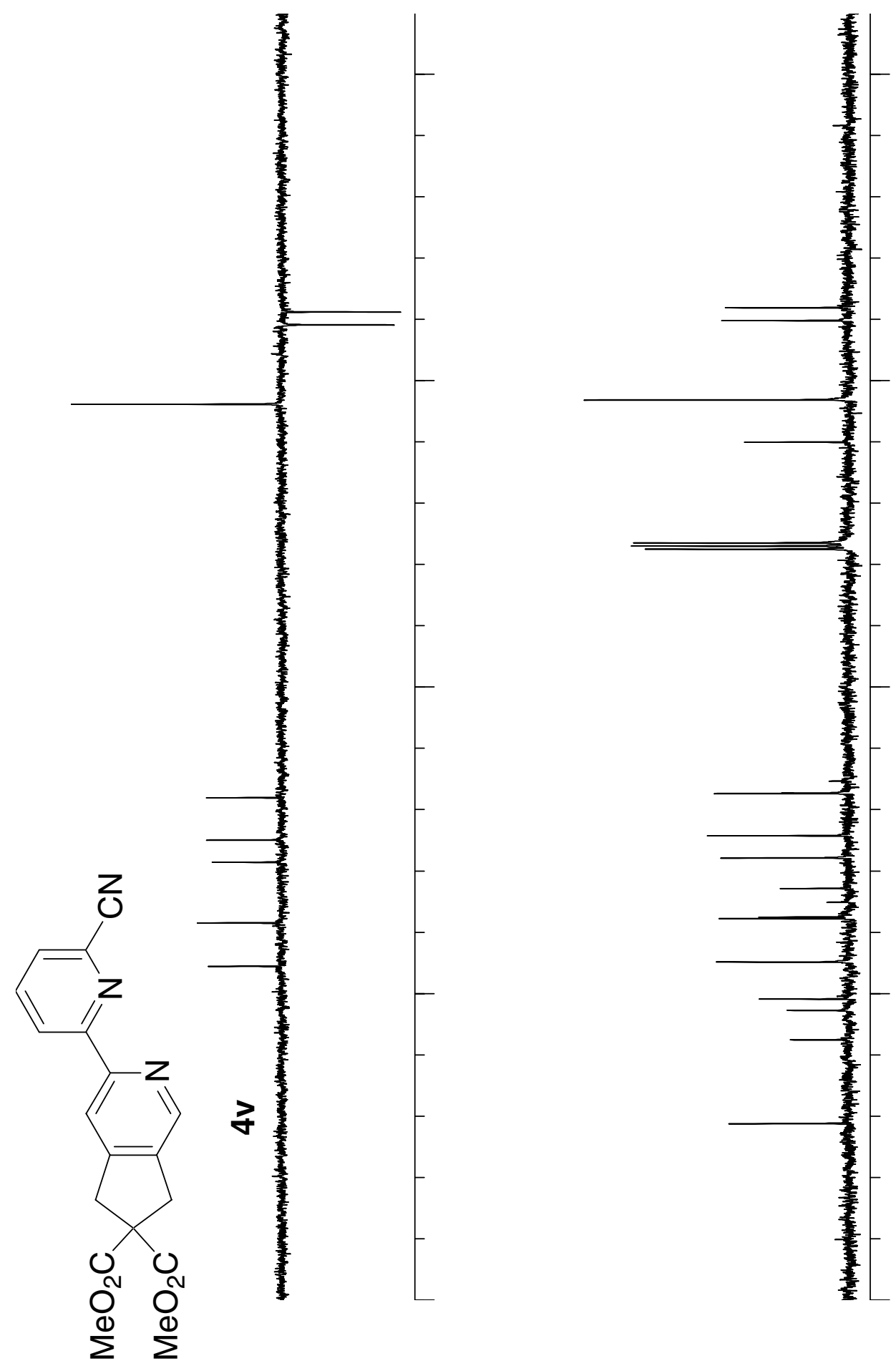
$\bar{\nabla}$

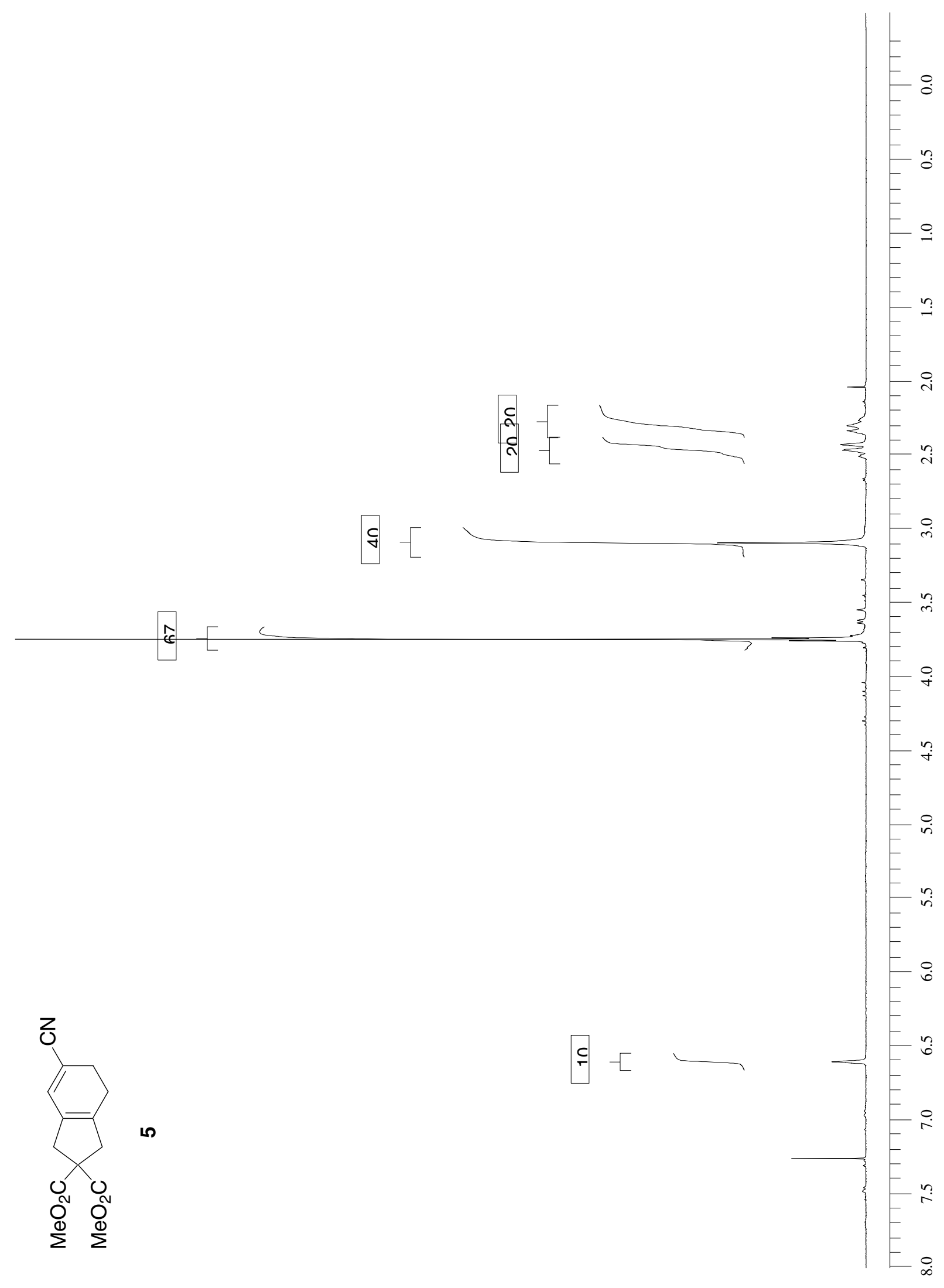


$\widetilde{N}$
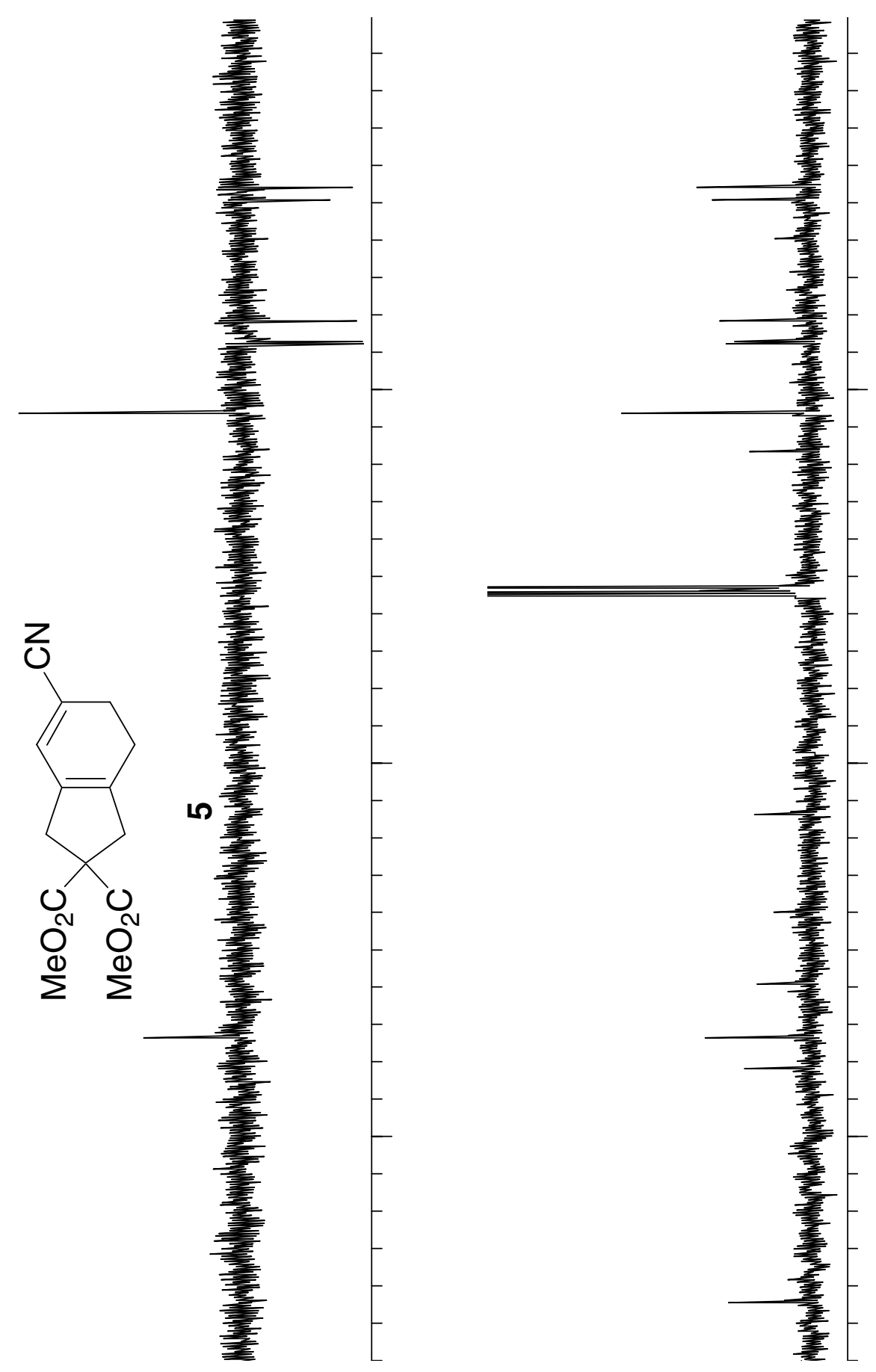


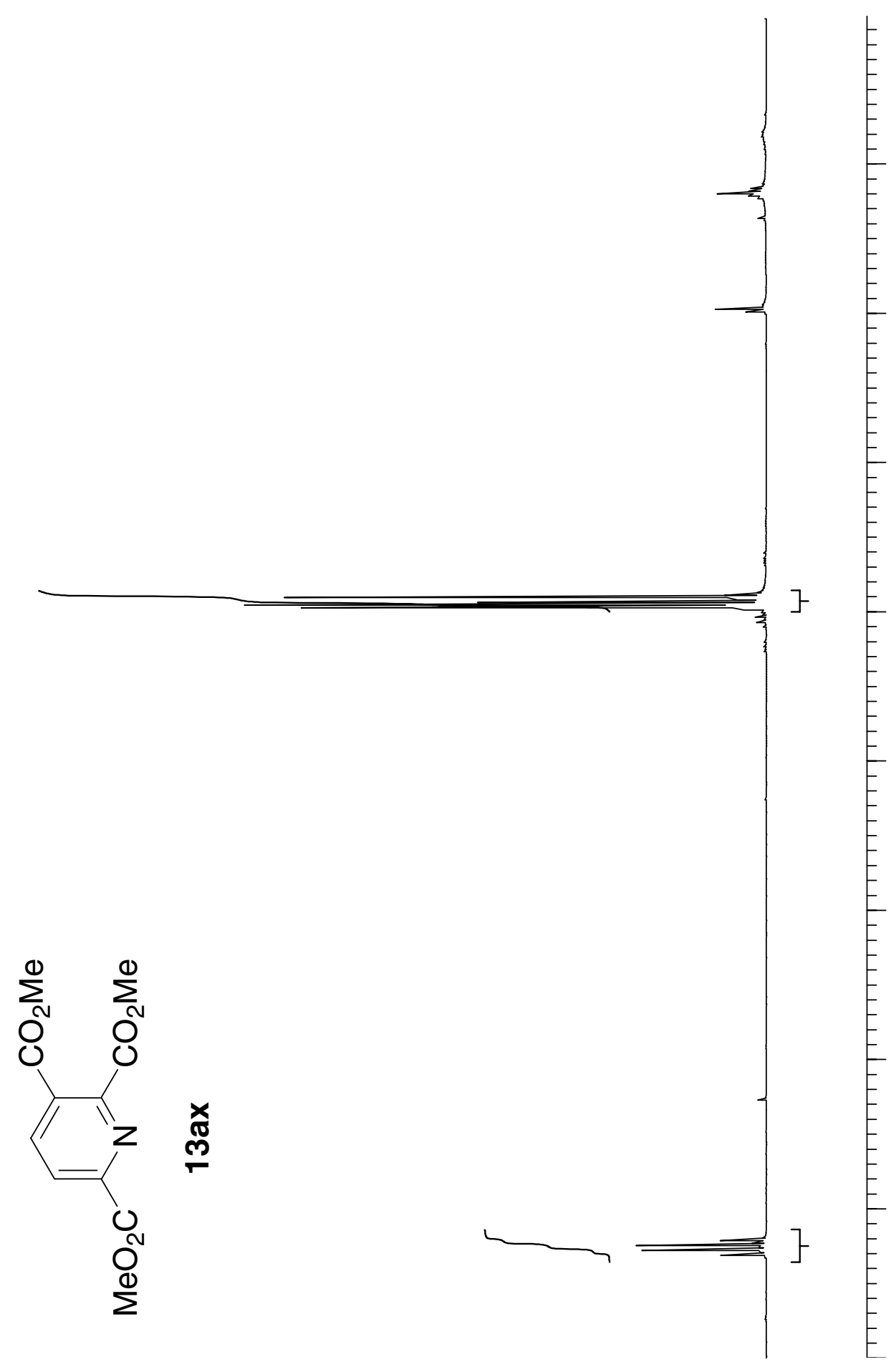




$$
\text { 복 }
$$




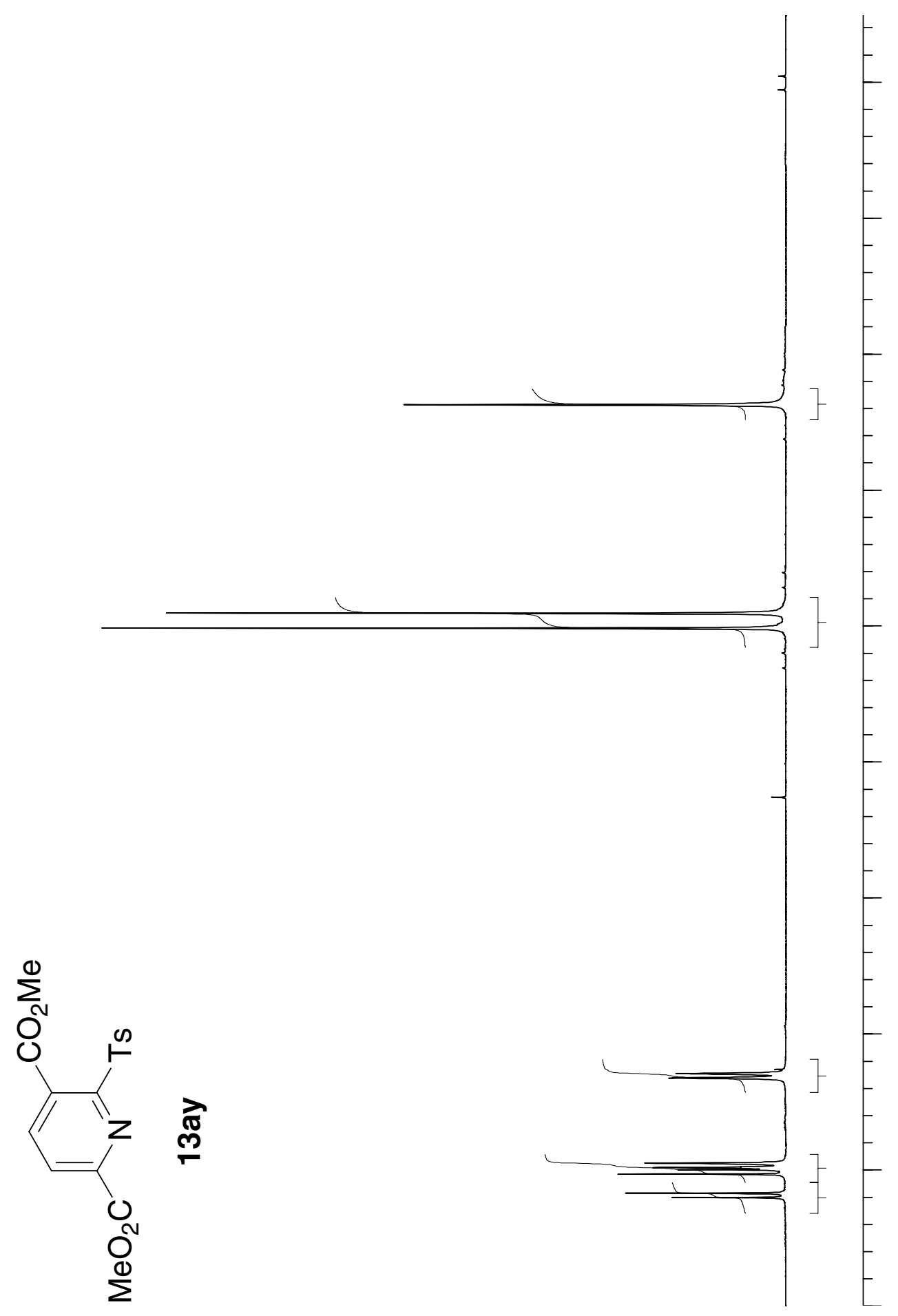


๕ั
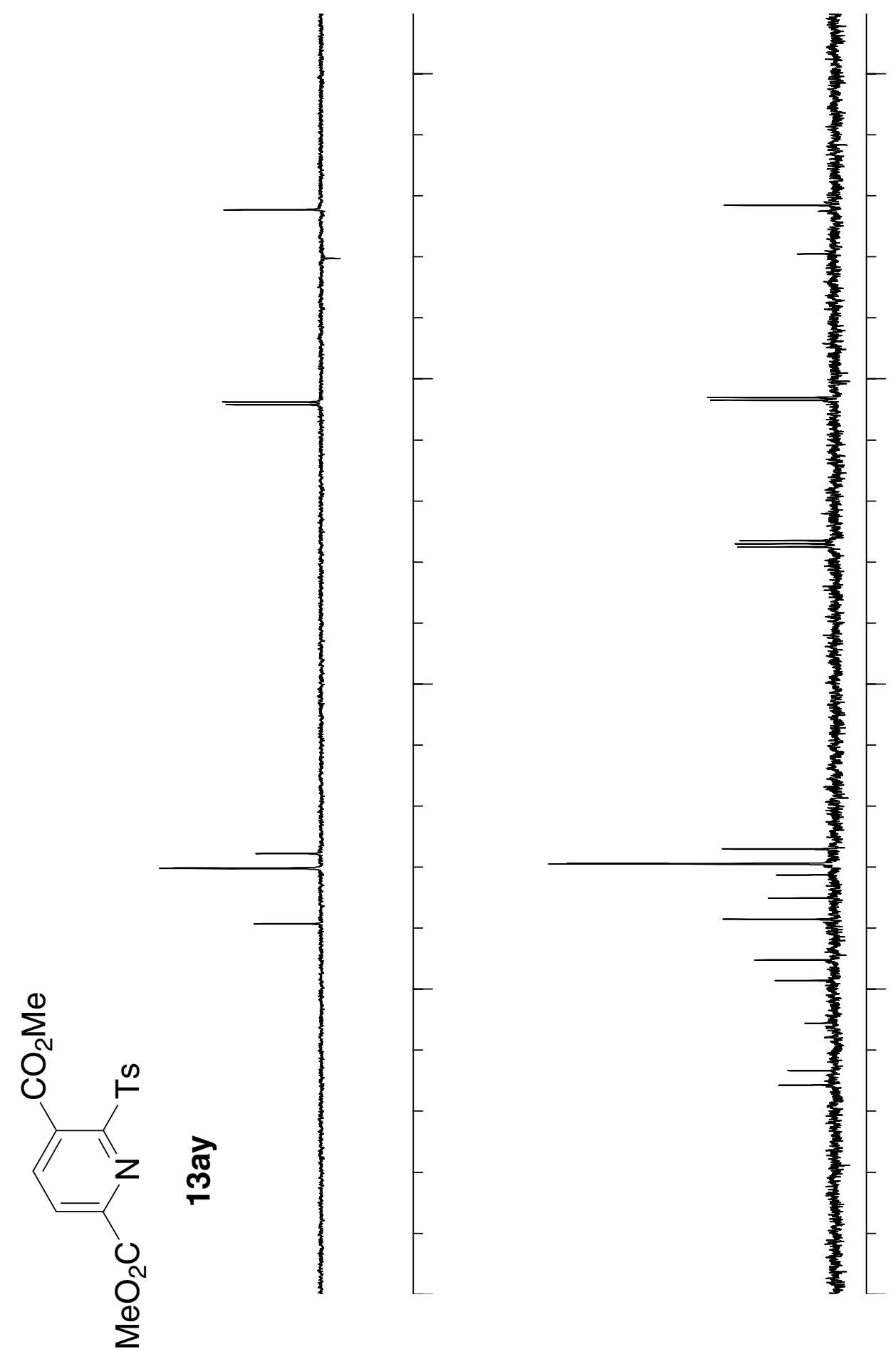
훙

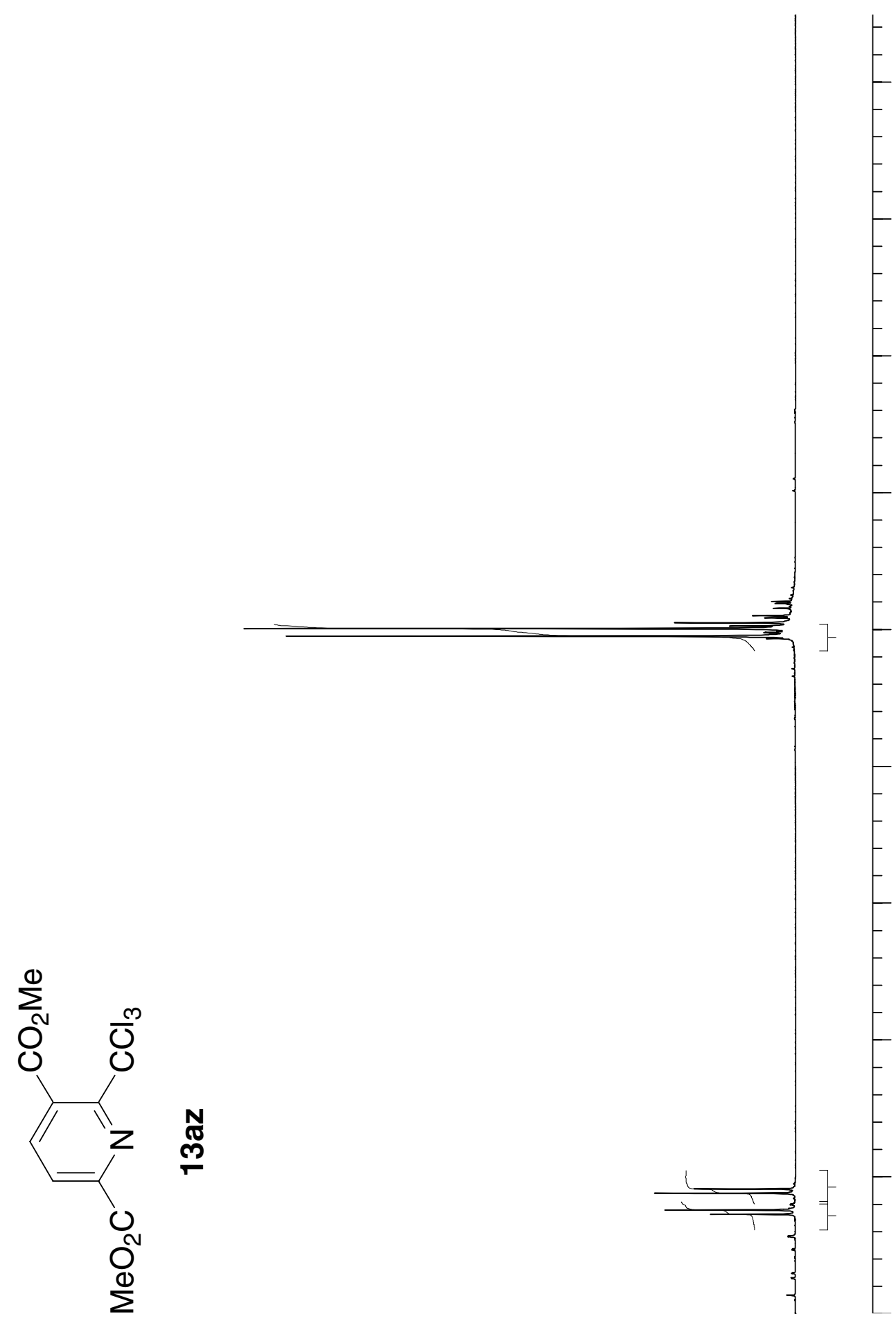




$$
H
$$




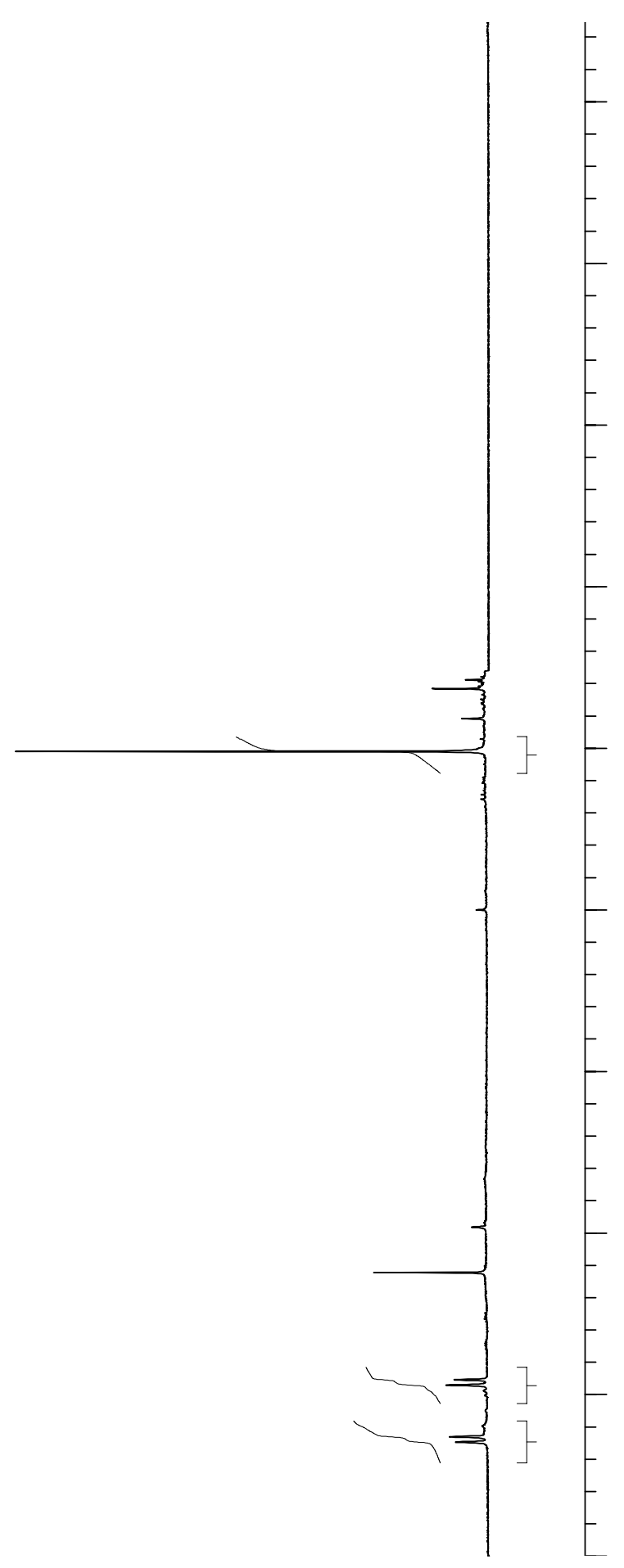

